\title{
Accelerating fatigue simulations of a phase-field damage model for rubber
}

\author{
Pascal J. Loew ${ }^{\mathrm{a}, \mathrm{b}, *}$, Leong H. Poh ${ }^{\mathrm{c}}$, Bernhard Peters ${ }^{\mathrm{a}}$, Lars A.A. Beex \\ ${ }^{a}$ Faculty of Science, Technology and Medicine, University of Luxembourg \\ ${ }^{b}$ SISTO Armaturen S.A., Echternach \\ ${ }^{c}$ Department of Civil and Environmental Engineering, National University of Singapore
}

\begin{abstract}
Phase-field damage models are able to describe crack nucleation as well as crack propagation and coalescence without additional technicalities, because cracks are treated in a continuous, spatially finite manner. Previously, we have developed a phase-field model to capture the rate-dependent failure of rubber, and we have further enhanced it to describe failure due to cyclic loading. Although the model accurately describes fatigue failure, the associated cyclic simulations are slow. Therefore, this contribution presents an acceleration scheme for cyclic simulations of our previously introduced phase-field damage model so that the simulation speed is increased to facilitate large-scale simulations of industrially relevant problems. We formulate an explicit and implicit cycle jump method, which, depending on the selected jump size, reduces the calculation time up to $99.5 \%$. To circumvent the manual tuning of the jump size, we also present an adaptive jump size selection procedure. Thanks to the implicit adaptive scheme, all material parameters are identified from experiments, which include fatigue crack nucleation and crack growth. Finally, the model and its parameters are validated with additional measurements of the fatigue crack growth rate.
\end{abstract}

Keywords: Phase-field, fatigue, damage, rubber, cycle jump

\footnotetext{
* Corresponding author

Email address: loew.pascal@gmail.com (Pascal J. Loew)
}

Preprint submitted to Computer Methods in Applied Mechanics and Engineering, July 3, 2020 


\section{Introduction}

Numerous structural components and applications are subjected to cyclic loading. Accurate simulations to identify the cycle-to-failure number not only reveal the best design but also reduce maintenance efforts and associated costs. Fatigue simulations require both a failure model able to accumulate damage due to repetitive loading and fast solution strategies for cyclic loading ([1]).

Treating cracks in a continuous manner with a finite damage zone controlled by a length scale parameter, phase-field damage models are able to handle effortless topological complex cracks. They do not require additional enhancements to describe crack nucleation, branching and crack coalescence (2], 3], 4]), and the location of cracks needs not to be known in advance, as for instance with standard cohesive zone models ([5], [6], [7], [8]). We want to note that coupling the extended finite element method (XFEM, 9, 10, 11]) with a cohesive zone model can deal with cracks not known in advance ([12]).

Phase-field models are similar to gradient-enhanced damage models ([13, [14, [15]), as both rely on a length scale parameter to obtain mesh independent results (16], [17, [18]). Miehe and Schänzel [19] were the first to extend phasefield damage models to finite strains and rubber, while its extension to ratedependency, with experimental identification and validations can be found in 20]. The ability to represent complex crack patterns and coalescence was highlighted by Wu et al. 21], who applied the method to carbon black reinforced rubber. San and Waisman [22] and Russ and Waisman 23] are other examples in which phase-field damage models were applied - particularly to optimize structural designs.

Focusing on cyclic loading, Amendola et al. 24] presented a fatigue phase-field damage model by introducing a fatigue history variable in the Ginzburg-Landau equation. Caputo and Fabrizio [25] also considered an accumulation of damage dependent on the time and the environment (i.e. aging). Boldrini et al. 26] equipped a phase-field damage model for fatigue with temperature effects due to the load cycles. Alessi et al. [27] presented a different approach, which reduces 
the fracture toughness with an increase of the load cycle. While Alessi et al. 27. focused exclusively on one-dimensional examples, Carrara et al. 28, and Mesgarnejad et al. 29] proposed similar models for two- and three-dimensional multi-crack configurations. Lo et al. 30] defined viscous damage dissipation so that crack growth under cyclic loading occurs. Loew et al. 1] presented a model for finite strains and validated two-dimensional simulations with experimental results.

Many contributions focus on the acceleration of phase-field fracture simulations. Heister et al. 31] proposed adaptive mesh refinement schemes, Cheng et al. [32] introduced a wavelet-enriched adaptive hierarchical framework and Patil et al. 33] combined phase-field damage modeling with XFEM. All three works shorten the computing time, since a fine mesh is only required in the vicinity of the crack. Another possibility is to use a monotonic solver, so that the elaborate iterations in the conventionally applied staggered scheme ([2], [34]) between the coupled damage-displacement equations are avoided ([35]). Although all these methods reduce the computational effort, the application to cyclic loading is still not practical, since every load cycle must be fully simulated.

The aim of this contribution is, therefore, to redevelop explicit and implicit cycle jump schemes from literature for fatigue phase-field simulations. We adopt the acceleration scheme for our earlier fatigue phase-field model of [1, modified here to incorporate a new fatigue damage source.

The first cycle jump scheme was introduced for continuum damage models by Van Paepegem et al. 36] to extrapolate the stiffness degradation of composites. Turon et al. [37] used this idea to simulate high cycle delamination with cohesive elements and Cojocaru et al. 38 extrapolated both the amount of damage as well as other state variables. Peerlings et al. 39. introduced a predictorcorrector cycle jump scheme for high-cycle fatigue to enable large cycle jumps. Decomposing the problem into slow (averaged) and fast (oscillation inside a load cycle) time components, two time-scale asymptotic time integration for fatigue problems was presented by Oskay and Fish [40, which Heczko and Kottner [41] applied to elastomeric materials. Since this multi time-scale approach requires 
the solution of two subproblems at each time-step, the speed may not be much improved compared to that of single time-scale calculation ([42]).

Joseph et. al 43 proposed a wavelet-based transformation with an implicit cycle jump scheme. In contrast to standard finite element simulations of solids, the wavelet framework does not solve for the nodal displacements at each time increment, but for the coefficients of the wavelets, which form the nodal displacements at all time instances for one load cycle. Comparing a standard finite element implementation to the wavelet-based implementation of Joseph et al. [43], both using an implicit cycle jump scheme, Beusink [44] showed that most of the speed up is related to the implicit scheme and not the wavelet transformation. Accordingly, we focus only on cycle jump schemes in this work.

Besides the main aim of proposing explicit and implicit cycle jump schemes to accelerate fatigue phase-field simulations, this contribution contains three additional novelties:

- A new (rate-independent) fatigue damage source is introduced in the framework of Loew et al. [1, which prohibits fatigue crack growth due to compressive stresses.

- Identification and validation experiments, including crack nucleation and growth, are presented.

- An adaptive jump size procedure is formulated for the implicit scheme in order to circumvent the need for manual tuning of the step size for cycle jumps.

The paper is organized as follows. In Chapter 2, we present the fatigue phasefield damage model for rubber. Following, we introduce the explicit and implicit cycle jump schemes to accelerate the calculations in Chapter 3, and demonstrate the performance of both schemes in Chapter 4 . Chapter 5 presents the experimental validation and we conclude the contribution in Chapter 6 .

We denote scalars with lowercase and capital letters $(a$ and $A$ ). Vectors are written in bold (a, A). 


\section{Fatigue phase-field damage model}

In this section, we present our fatigue phase-field damage model. Damage (i.e. cracks) are described with a scalar variable $d \in[0,1]$ so that $d=1$ on an internal discontinuity $\Gamma_{0}$ and $d=0$ on $\Omega_{0} \backslash \Gamma_{0}$ (Fig. 1 a)).

a)

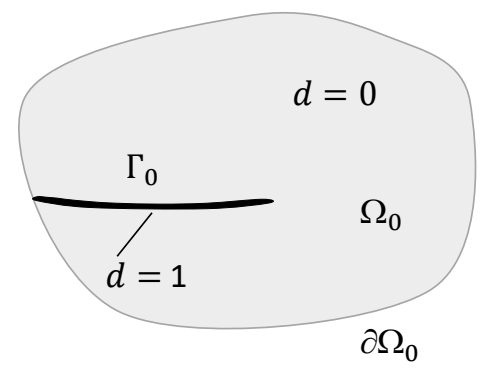

b)

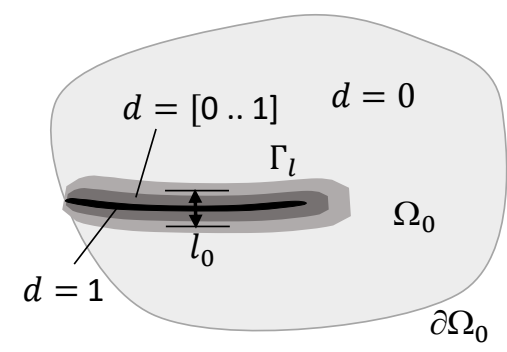

Figure 1: Fatigue phase-field damage model in 2D: A sharp crack $\Gamma_{0}(\mathrm{a})$ is approximated with a crack surface $\Gamma_{l}(\mathrm{~b})$. The length scale $l_{0}$ controls the width of the process zone.

We describe the motion and deformation of the body $\Omega_{0}$ in the reference configuration by displacement $\mathbf{u}$ and deformation gradient $\mathbf{F}=\mathbf{I}+\nabla_{0} \mathbf{u}$. I denotes the unit tensor and spatial derivatives associated with the reference configuration are denoted by $\frac{\partial \cdot}{\partial \mathbf{X}}=\nabla_{0}(\cdot)$. The balance of mechanical energy requires:

$$
\dot{E}+\dot{D}-\dot{R}-\dot{P}^{e x t}=0,
$$

where $\dot{E}, \dot{D}$ and $\dot{P}^{e x t}$ denote the internally stored energy, dissipated energy and the externally supplied energy per time unit, respectively. Fatigue damage is, on a pure phenomenological basis, described by fatigue damage source $\dot{R}$ ([1]). $\dot{R}$ could also be interpreted as a reduction of $\dot{D}$. That would correspond to recently published fatigue phase-field damage models ([27, [28] and [29]), where a reduction of the dissipated energy is achieved by multiplication with a load history dependent variable. However, we prefer the interpretation of $R$ as the energy of an extrinsic volumetric micro-force (see [45, [46, 44], 48] and 49]). According to Frémond and Nedjar ([46]) such a source of damage can for exam- 
ple be produced by chemical or electrical actions, which break the bonds inside a material. We assume that cyclic loading leads to stress and strain-induced aging processes, i.e. the cross-link density evolves due to chain-scission and cross-linking reactions. Following, microcracks form, merge and cause damage at the macroscale. In this case, extrinsic does not refer to a global external force, but to processes on a different scale $1^{1}$ The relations for $\dot{E}, \dot{D}, \dot{P}^{e x t}$ and $\dot{R}$ are presented in the following subsection.

\subsection{Rate of internally stored energy}

The internally stored energy in the bulk reads:

$$
E=\int_{\Omega_{0} \backslash \Gamma_{0}} \psi^{\text {elas }} d V
$$

with strain energy density $\psi^{\text {elas }} 2$ By introducing degradation function $g_{d}=$ $g_{d}(d)$, the integration in Eq. (2) can be performed over the entire domain $\Omega_{0}$ :

$$
E=\int_{\Omega_{0}}\left(g_{d} \psi^{\text {elas }}\right) d V
$$

The degradation function controls the mechanical response with respect to the virgin state and requires:

$$
\begin{aligned}
& g_{d}(d=0)=1 \\
& g_{d}(d=1)=0 \\
& \left.\frac{\partial g_{d}}{\partial d}\right|_{d=1}=0 .
\end{aligned}
$$

We use a recently proposed degradation function ([50], [51]):

$$
g_{d}=\frac{(1-d)^{2}}{(1-d)^{2}+a d\left(1-\frac{1}{2} d\right)},
$$

where $a$ denotes an additional material parameter. Wu and Nguyen [50] derived from 1D analytical results that the failure strength $f_{t} \propto\left(a \cdot l_{0}\right)^{-1 / 2}$. Therefore,

\footnotetext{
${ }^{1}$ This interpretation enables us to imply that the rate of dissipated crack energy is independent of the (fatigue) load history variable $h$ (Eq. 16). Or in other words, a crack caused by cyclic loading dissipates as much energy as a crack caused by monotonic loading.

${ }^{2}$ Rate-dependent effects could be incorporated by splitting the strain energy density into an elastic and viscous contribution $([20])$.
} 
by adjusting material parameter $a$ according the value of length scale $l_{0}$, the failure strength and the force-displacement responses can be made independent of the length scale ([1]).

The rate of the internally stored energy then reads:

$$
\dot{E}=\int_{\Omega_{0}}\left(g_{d} \frac{\partial \psi^{\text {elas }}}{\partial \mathbf{F}}: \dot{\mathbf{F}}+\frac{\partial g_{d}}{\partial d} \psi^{\text {elas }} \dot{d}\right) d V,
$$

and the elastic strain energy density is defined according to [52]:

$$
\psi^{\text {elas }}=\sum_{i=1}^{3} C_{i}\left(I_{1}-3\right)^{i},
$$

where $I_{1}=\operatorname{tr}\left(\mathbf{F}^{T} \cdot \mathbf{F}\right)$ and $C_{i}$ denote the hyperelastic material parameters. The first Piola-Kirchhoff stress can then be expressed as follows:

$$
\mathbf{P}=\frac{\partial \psi^{\text {elas }}}{\partial \mathbf{F}}=2 \sum_{i=1}^{3} i C_{i}\left(I_{1}-3\right)^{(i-1)} \mathbf{F}
$$

\subsection{Rate of dissipation}

Since we neglect rate-dependent effect, the rate of dissipation is only attributed to the rate of dissipation due to crack growth:

$$
\dot{D}=\dot{D}^{\text {crack }}
$$

As the energy dissipated by the formation of a unit crack area is denoted by $G_{c}$, the energy dissipated through crack growth is expressed as:

$$
D^{\text {crack }}=\int_{\Gamma_{0}} G_{c} d A
$$

The sharp discontinuity $\Gamma_{0}$ is approximated $\Gamma_{0} \approx \Gamma_{l}=\int_{\Omega_{0}} \gamma_{l} d V$ with crack density function $\gamma_{l}=\gamma_{l}\left(d, l_{0}\right)$ (4 $)$, which introduces length scale $l_{0}$ to control the width of the damage zone (see Fig. 1 b)). Thus, multiplying Eq. 10 with $\gamma_{l}$, we avoid integrating over the fractured surface and change the integration to a volume integral over the domain $\Omega_{0}$ :

$$
D^{\text {crack }}=\int_{\Omega_{0}} G_{c} \gamma_{l} d V
$$


Following [51, we define the crack density function:

$$
\gamma_{l}=\frac{1}{c_{0}}\left[\frac{1}{l_{0}} w+l_{0}\left(\nabla_{0} d \cdot \nabla_{0} d\right)\right],
$$

where $w$ denotes a geometric crack function $w=w(d) \in[0,1]$ with the constraints $w(d=0)=0$ and $w(d=1)=1 . \quad c_{0}$ denotes a scaling parameter to ensure $\Gamma_{0}=\Gamma_{l}$ for $l_{0} \rightarrow 0$. Defining $w=2 d-d^{2}$ ([50]), we write $c_{0}=4 \int_{0}^{1} \sqrt{w(\delta)} d \delta=\pi$.

By differentiating with respect to time, the dissipation rate due to crack formation reads:

$$
\dot{D}^{\text {crack }}=\int_{\Omega_{0}} G_{c} \frac{1}{\pi}\left(\frac{1}{l_{0}}(2-2 d) \dot{d}+2 l_{0} \nabla_{0} d \cdot \nabla_{0} \dot{d}\right) d V .
$$

The second law of thermodynamics requires:

$$
\dot{D}=\dot{D}^{\text {crack }} \geq 0
$$

which is fulfilled by imposing constraint $\dot{d} \geq 0$ (20]).

\subsection{Rate of externally applied energy}

By denoting the surface traction and volumetric body force vector $\mathbf{t}_{0}$ and $\mathbf{b}_{0}$, the rate of externally applied energy is:

$$
\dot{P}^{e x t}=\int_{\partial \Omega_{0}} \mathbf{t}_{0} \cdot \dot{\mathbf{u}} d A+\int_{\Omega_{0}} \mathbf{b}_{0} \cdot \dot{\mathbf{u}} d V .
$$

\subsection{Fatigue damage source}

First, we introduce $h=h(t)$, an accumulated history variable, which cannot decrease:

$$
\dot{h} \geq 0 .
$$

$h$ is defined later but basically any scalar quantity, which is able to describe fatigue loads, may be used. The energetic contribution of fatigue damage reads:

$$
R=\int_{\Omega_{0}} \int_{T} g_{f} \zeta_{d} h^{\zeta_{e}} \dot{d} d t d V
$$


where $g_{f}$ denotes a fatigue degradation function, and $\zeta_{d}$ and $\zeta_{e}$ two fatigue material parameters. Fatigue failure of rubbers can often be described with a power law (see Figs. 16, 20 and 23), so that the formulation in Eq. (17) appears natural. The influence of these material parameters is presented in Appendix A. Note that this is a purely phenomenological formulation, primarily based on macroscopic experimental observations. "Work" is only performed when damage grows, so $\dot{d}$ is included. It is also worth mentioning that $R$ is a processdependent variable and not a state function.

A load history $h=h(t, \epsilon)$ with $\epsilon$ denoting the strain was presented by Alessi et. al [27. However, Carrara et al. 28] demonstrated that this leads to mesh dependent results because of strain singularities at crack tips and proposed instead an energetic formulation. In our rate-dependent framework ([1]), promising results were achieved by basing $h=h\left(t, D^{v i s c}\right)$ on the viscous dissipation of the bulk $D^{\text {visc }}$.

Departing from [1, we neglect rate-dependent effects and propose a load history variable $h=h\left(t, \psi^{\text {elas }}\right)$ depending on the elastic strain energy density $\psi^{\text {elas }}=\psi^{\text {elas }}\left(I_{1}\right){ }^{3}$ Rate-dependent effects play a major role in fracture of rubber ([20, 53], 54]) and for high frequent cyclic loading heat build-up, followed by temperature depending change of the material properties and thermal degradation can be experimentally observed ([55], [56]). However, Stadlbauer et al. [57] also showed that the influence of the strain rate and the load function (e.g. sine wave or "square" pattern) on the service life in temperature controlled experiments is insignificant. We limit ourselves to relatively low strain-rates to ensure temperature constancy in our experiments.

The load history is:

$$
h=\frac{1}{\hat{h}} \int_{T} s_{l} s_{c} \dot{\psi}^{\text {elas }} d t
$$

where $s_{l}$ and $s_{c}$ denote two switch functions $\hat{4}=1 \mathrm{~N} / \mathrm{mm}^{2}$ denotes a normalization parameter to ensure $h$ is dimensionless. Without this factor, units

\footnotetext{
${ }^{3} I_{1}=\operatorname{tr}\left(\mathbf{F}^{T} \cdot \mathbf{F}\right)$ is the first invariant of the right Cauchy-Green tensor.
} 
would not match if material parameter $\zeta_{e} \neq 1$. The first switch, $s_{l}$, ensures that damage growth only occurs due to loading, and not due to unloading:

$$
s_{l}= \begin{cases}1, & \text { if } \dot{I}_{1} \geq 0 \\ 0, & \text { otherwise }\end{cases}
$$

The second switch, $s_{c}$, prevents fatigue damage growth due to compressive loading and accelerates fatigue crack growth in regions with a high hydrostatic pressure $p=\frac{1}{3} \operatorname{tr}\left(\mathbf{F P}^{T}\right):{ }^{5}$

$$
s_{c}= \begin{cases}1+\zeta_{p} \cdot \max \left(p-\zeta_{t}, 0\right), & \text { if } p \geq 0 \\ 0, & \text { otherwise }\end{cases}
$$

$\zeta_{p}$ and $\zeta_{t}$ denote two additional fatigue material parameters to be defined. In rubber, (fatigue) cracks tend to nucleate in regions of high hydrostatic pressure (48], [59], 60] and [61]) and we take this effect into account with $\zeta_{t}$ and $\zeta_{p}$. A demonstration of the characteristics of the new load history $h$ can be found in Appendix B

Fatigue degradation function $g_{f}=g_{f}(d)$ has the following properties:

$$
\begin{aligned}
& g_{f}(d=0)=1 \\
& g_{f}(d=1)=0 .
\end{aligned}
$$

It ensures that no work is performed in completely damaged domains. As in [1, we set $g_{f}=-\frac{\partial g_{d}}{\partial d}$, where $g_{d}$ denotes the degradation function of the bulk, see Eq. (5). With this choice, $h$ and $\psi^{\text {elas }}$ are similarly reduced with an increase of the damage (see Eq. (26)), leading to a relatively straightforward implementation.

\footnotetext{
${ }^{4} \mathrm{~A}$ third switch $s_{n}$ could be additionally introduced to ensure that fatigue damage can only grow when a threshold $\left(\zeta_{n}\right)$ is reached:

$$
s_{n}= \begin{cases}1, & \text { if } I_{1} \geq \zeta_{n} \\ 0, & \text { otherwise }\end{cases}
$$

Gent [58] shows the experimental justification to use such a threshold for rubber, but since we do not have experimental data at these load levels we do not include this third switch. ${ }^{5}$ Definition of $p$ according to 66].
} 
Finally, taking the time derivatives of Eq. [17), the fatigue damage source reads:

$$
\dot{R}=\int_{\Omega_{0}}-\frac{\partial g_{d}}{\partial d} \zeta_{d} h^{\zeta_{e}} \dot{d} d V
$$

\subsection{Balance of mechanical energy}

Inserting Eqs. (6), (9), (15) and (22) into Eq. (1), we obtain:

$$
\begin{aligned}
& -\int_{\Omega_{0}}\left(\nabla_{0} \cdot\left(g_{d} \frac{\partial \psi^{\text {elas }}}{\partial \mathbf{F}}\right)+\mathbf{b}_{0}\right) \cdot \dot{\mathbf{u}} d V \\
& +\int_{\partial \Omega_{0}}\left(\left(g_{d} \frac{\partial \psi^{\text {elas }}}{\partial \mathbf{F}}\right) \cdot \mathbf{n}_{0}-\mathbf{t}_{0}\right) \cdot \dot{\mathbf{u}} d A \\
& +\int_{\Omega_{0}}\left(\frac{\partial g_{d}}{\partial d}\left(\psi^{\text {elas }}+\zeta_{d} h^{\zeta_{e}}\right)+\frac{G_{c}}{l_{0} \pi}(2-2 d)-G_{c} l_{0} \frac{2}{\pi} \nabla_{0}^{2} d\right) \dot{d} d V \\
& +\int_{\partial \Omega_{0}} G_{c} l_{0} \frac{2}{\pi} \nabla_{0} d \cdot \mathbf{n}_{0} \dot{d} d A=0 .
\end{aligned}
$$

With the following Neumann boundary conditions:

$$
\left(g_{d} \frac{\partial \psi^{\text {elas }}}{\partial \mathbf{F}}\right) \cdot \mathbf{n}_{0}=\mathbf{t}_{0} \text { and } \nabla_{0} d \cdot \mathbf{n}_{0}=0
$$

we extract the governing equation for displacement field $\mathbf{u}$ :

$$
\nabla_{0} \cdot\left(g_{d} \frac{\partial \psi^{\text {elas }}}{\partial \mathbf{F}}\right)+\mathbf{b}_{0}=0
$$

and for damage field $d$ :

$$
\frac{\partial g_{d}}{\partial d}\left(\psi^{\text {elas }}+\zeta_{d} h^{\zeta_{e}}\right)+\frac{G_{c}}{l_{0} \pi}(2-2 d)-G_{c} l_{0} \frac{2}{\pi} \nabla_{0}^{2} d=0 .
$$

\subsection{Tension-compression split}

To account for the closing of cracks and to prevent crack growth due to compressive loading ([62], 63]), we split the bulk energy into a positive $\psi^{+}$(tensile) and negative $\psi^{-}$(compression) part ([3], 64] $)$[6

(Nearly) incompressible rubbery polymers are described with an elastic strain energy density decomposed into an isochoric and volumetric part ([65]):

$$
\psi^{\text {elas }}=\psi^{\text {iso }}(\overline{\mathbf{F}})+\psi^{\text {vol }}(J)
$$

\footnotetext{
${ }^{6}$ Switch $s_{c}$ (Eq. 20 prohibits fatigue crack growth in compression. The split of the energy does not stop $h$ from growing because $h=h\left(\psi^{\text {elas }}\right)$.
} 
where $\overline{\mathbf{F}}=J^{-1 / 3} \mathbf{F}$ and $J=\operatorname{det}(\mathbf{F})$. In case of plane stress situations (which we restrict ourselves to), the incompressibility constraint is generally incorporated via substitution ([6]), so that $J=1, \psi^{\text {vol }}=0$ and $\psi^{\text {elas }}=\psi^{\text {iso }}$. Using hydrostatic pressure $p$ to distinguish between compression and tension, we write 7

$$
\begin{aligned}
& \psi^{+}= \begin{cases}\psi^{\text {elas }}, & \text { if } p \geq 0 \\
0, & \text { otherwise }\end{cases} \\
& \psi^{-}= \begin{cases}0, & \text { if } p \geq 0 \\
\psi^{\text {elas }}, & \text { otherwise. }\end{cases}
\end{aligned}
$$

Application of this split ([3, 64]) in Eq. 25) and Eq. 26) leads to:

$$
\nabla_{0} \cdot\left(g_{d} \frac{\partial \psi^{+}}{\partial \mathbf{F}}+\frac{\partial \psi^{-}}{\partial \mathbf{F}}\right)+\mathbf{b}_{0}=0
$$

and:

$$
\frac{\partial g_{d}}{\partial d}\left(\psi^{+}+\zeta_{d} h^{\zeta_{e}}\right)+\frac{G_{c}}{l_{0} \pi}(2-2 d)-G_{c} l_{0} \frac{2}{\pi} \nabla_{0}^{2} d=0 .
$$

Eq. (29) and Eq. (30) are then transformed into their respective weak form using the standard Galerkin procedure. We discretize the 2D domains under plane stress circumstances with bilinear isoparametric quadrilateral elements. Details on the numerical implementation can be found in 20 .

Since we limit ourselves to plane stress applications, practically only thin structures can be simulated. The ability of these structures to absorb compressive stress is low because they buckle quickly in practice. Therefore, a comprehensive study of compressive loads is not realistic and we do not claim that the proposed split is advantageous over recently published works, see e.g. 67. or 68. Still, we demonstrate in Appendix D that the proposed split successfully avoids crack penetration and crack growth in compression.

\footnotetext{
${ }^{7}$ For the present manuscript, we limit ourselves to incompressible materials in plane stress situations and the material model of Yeoh [52. The boundary conditions $P_{33}=0$ and $J=1$ enable the direct calculation of $p=2 \frac{\partial \psi^{\text {elas }}}{\partial I_{1}}\left(\frac{1}{3} I_{1}-F_{33}^{2}\right)$ (see $\underline{66}$ for details). Since $\frac{\partial \psi^{\text {elas }}}{\partial I_{1}} \geq 0$, the split could equally depend on the deformation measure $\left(\frac{1}{3} I_{1}-F_{33}^{2}\right)$.
} 
In plane stress settings the incompressibility constraint is incorporated by substitution - instead of via (pressure) fields of lagrange multipliers for perfect incompressibility, or via penalty terms for near incompressibility. Therefore, the standard volumetric deformation based split (64) is not applicable since $J=1$ and we do not need to compute the spectral decomposition of the strain tensor ([3]). 


\section{Explicit and implicit cycle scale time integration}

Lifetime predictions are accelerated thanks to explicit ([36], [37]) or implicit (43], 44]) cycle jump scheme. The basic idea is to simulate a few cycles, and then extrapolate the results for $\mathbf{u}, d$ and $h$ over a selected interval. Note that we do not only extrapolate the damage variable but all relevant variables ([37, [69, [70]). While the explicit extrapolation is solely based on past results, in the implicit scheme the values after the cycle jump are also considered. The implicit procedure is therefore comparable to an explicit procedure with a predictorcorrector step ([39]).

Depending on the extrapolation scheme and the non-linearity of the structural response, the jump size $\Delta N=1 . .10 . .10^{6}$ is fixed. The process of alternating between calculations and jumping of cycles is repeated until the lifetime of the component is reached.

This procedure reduces the number of simulated load cycles and consequently the total calculation time. First, we present the explicit scheme and compare it with the implicit scheme. Based on the first results in Section 4.1. we propose in Subsection 3.2 .2 an adaptive step size procedure. To ease the notation below, we store $\mathbf{u}, d$ (both nodal values) and $h$ (computed at the integration points) in $\mathbf{v}$.

\subsection{Explicit cycle scale time integration}

We extrapolate the evolution of variable $\mathbf{v}_{0, N}$ over a discrete number of load cycles $\Delta N$ with a second order Taylor series (see Fig,2). The index 0 indicates the start of a load cycle:

$$
\mathbf{v}_{0, N+\Delta N}=\mathbf{v}_{0, N}+\left.\Delta N \frac{\partial \mathbf{v}_{0}}{\partial N}\right|_{N}+\left.\frac{1}{2} \Delta N^{2} \frac{\partial^{2} \mathbf{v}_{0}}{\partial N \partial N}\right|_{N}
$$

with

and

$$
\left.\frac{\partial \mathbf{v}_{0}}{\partial N}\right|_{N}=\mathbf{v}_{0, N}-\mathbf{v}_{0, N-1}
$$

$$
\begin{aligned}
\left.\frac{\partial^{2} \mathbf{v}_{0}}{\partial N \partial N}\right|_{N} & =\left.\frac{\partial \mathbf{v}_{0}}{\partial N}\right|_{N}-\left.\frac{\partial \mathbf{v}_{0}}{\partial N}\right|_{N-1} \\
& =\mathbf{v}_{0, N}-2 \mathbf{v}_{0, N-1}+\mathbf{v}_{0, N-2}
\end{aligned}
$$




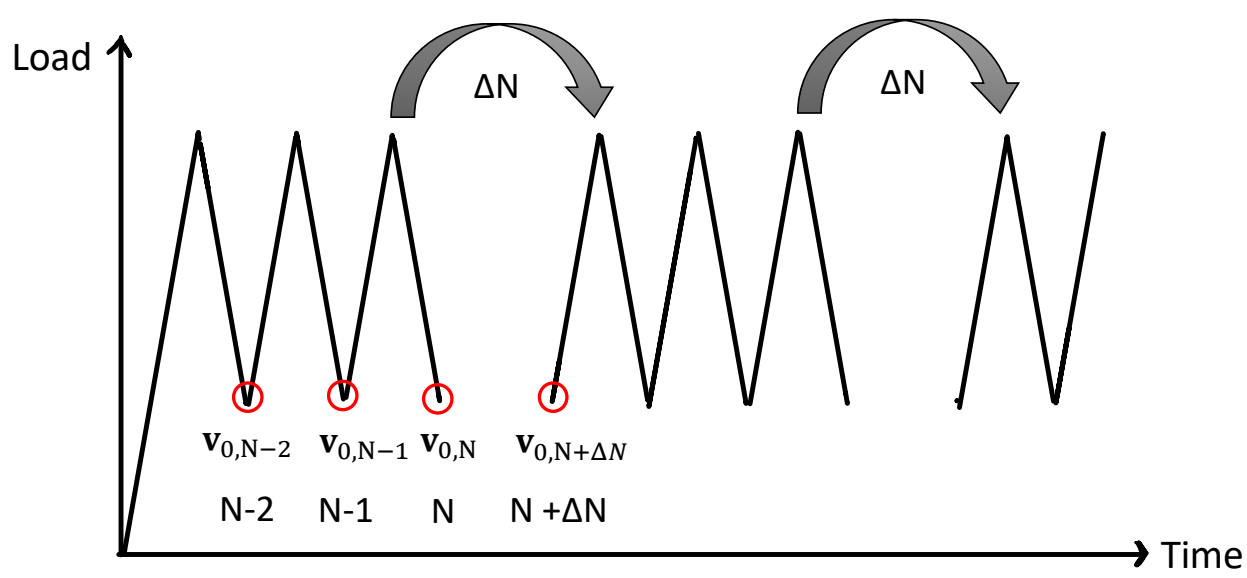

Figure 2: Explicit cycle jump scheme.

The algorithm of the explicit time integration is summarized in Algo. 1;

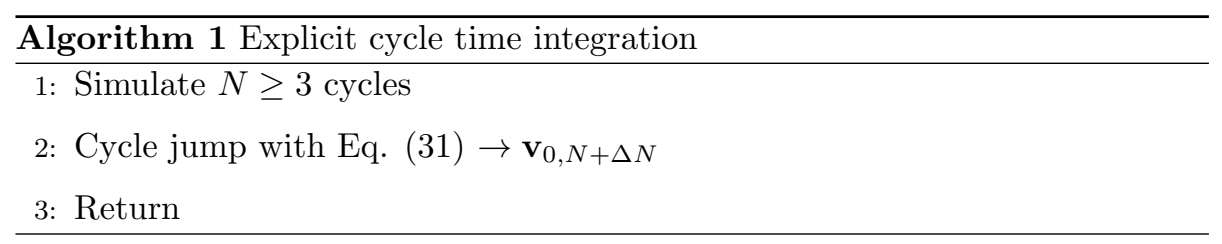

\subsection{Implicit cycle scale time integration}

The explicit extrapolation is solely based on past results. In the implicit scheme the values after the cycle jump are also considered $\left(\mathbf{v}_{0, N+\Delta N}\right.$ and $\left.\mathbf{v}_{q, N+\Delta N}\right)$, as displayed in Fig. 3. Index 0 indicates the start of a load cycle and index $q$ the end. Values at time steps in between are denoted by index $l$.

Following [44] we use a trapezoidal integration rule to extrapolate the variables:

$$
\mathbf{v}_{0, N+\Delta N}=\mathbf{v}_{0, N}+\frac{\Delta N}{2}\left(\left.\frac{\partial \mathbf{v}_{0}}{\partial N}\right|_{N+\Delta N}+\left.\frac{\partial \mathbf{v}_{0}}{\partial N}\right|_{N}\right),
$$

with

$$
\left.\frac{\partial \mathbf{v}_{0}}{\partial N}\right|_{N+\Delta N}=\mathbf{v}_{q, N+\Delta N}-\mathbf{v}_{0, N+\Delta N}
$$

and

$$
\left.\frac{\partial \mathbf{v}_{0}}{\partial N}\right|_{N}=\mathbf{v}_{q, N}-\mathbf{v}_{0, N}
$$


$\mathbf{v}_{q, N+\Delta N}$ and $\mathbf{v}_{0, N+\Delta N}$ are initially unknown. Therefore, we use a first order Taylor series to approximate:

$$
\mathbf{v}_{0, N+\Delta N}^{n}=\mathbf{v}_{0, N}+\left.\Delta N \frac{\partial \mathbf{v}_{0}}{\partial N}\right|_{N} .
$$

Exponent $n$ denotes the iteration of the implicit scheme (see Fig. 3). A full load cycle is then calculated so that $\mathbf{v}_{q, N+\Delta N}^{n}$ is also known. Eq. (34) leads to a non-linear equation:

$$
\begin{aligned}
\mathbf{f}^{i m p}= & \left(1+\frac{\Delta N}{2}\right) \mathbf{v}_{0, N+\Delta N}-\left(1-\frac{\Delta N}{2}\right) \mathbf{v}_{0, N} \\
& -\frac{\Delta N}{2}\left(\mathbf{v}_{q, N+\Delta N}+\mathbf{v}_{q, N}\right)=\mathbf{0},
\end{aligned}
$$

which we solve iteratively with the Newton-Raphson method:

$$
\begin{gathered}
\mathbf{v}_{0, N+\Delta N}^{n+1}=\mathbf{v}_{0, N+\Delta N}^{n}+\Delta \mathbf{v}_{0, N+\Delta N}^{n}, \\
\mathbf{f}^{i m p}+\left(\left.\frac{\partial \mathbf{f}^{i m p}}{\partial \mathbf{v}_{0}}\right|_{N+\Delta N}\right) \Delta \mathbf{v}_{0, N+\Delta N}^{n}=\mathbf{0} .
\end{gathered}
$$

Inserting Eq. 407 in Eq. (39):

$$
\mathbf{v}_{0, N+\Delta N}^{n+1}=\mathbf{v}_{0, N+\Delta N}^{n}-\left(\left.\frac{\partial \mathbf{f}^{i m p}}{\partial \mathbf{v}_{0}}\right|_{N+\Delta N}\right)^{-1} \mathbf{f}^{i m p} .
$$

The tangent matrix is:

$$
\left.\frac{\partial \mathbf{f}^{i m p}}{\partial \mathbf{v}_{0}}\right|_{N+\Delta N}=\left(1+\frac{\Delta N}{2}\right) \mathbf{I}-\left.\frac{\Delta N}{2} \frac{\partial \mathbf{v}_{q}}{\partial \mathbf{v}_{0}}\right|_{N+\Delta N} .
$$

The calculation of $\partial \mathbf{v}_{q} /\left.\partial \mathbf{v}_{0}\right|_{N+\Delta N}$ is discussed in more detail in Subsection 3.2 .1

Summarizing (see also Algo. 2), the implicit scheme starts with an explicit jump to estimate variables $\mathbf{v}_{0, N+\Delta N}^{n}$. A full load cycle is then simulated to obtain $\mathbf{v}_{q, N+\Delta N}^{n}$ and $\partial \mathbf{v}_{q}^{n} /\left.\partial \mathbf{v}_{0}\right|_{N+\Delta N}$. By application of Eq. 441, the initial estimation of the variables is updated $\mathbf{v}_{0, N+\Delta N}^{n+1}$. This process is repeated until the residual:

$$
\operatorname{Res}=\frac{\mathbf{f}^{i m p} \cdot \mathbf{f}^{i m p}}{\operatorname{length}\left(\mathbf{f}^{i m p}\right)},
$$

is smaller than Res $<$ Res $_{\text {target }}$. 


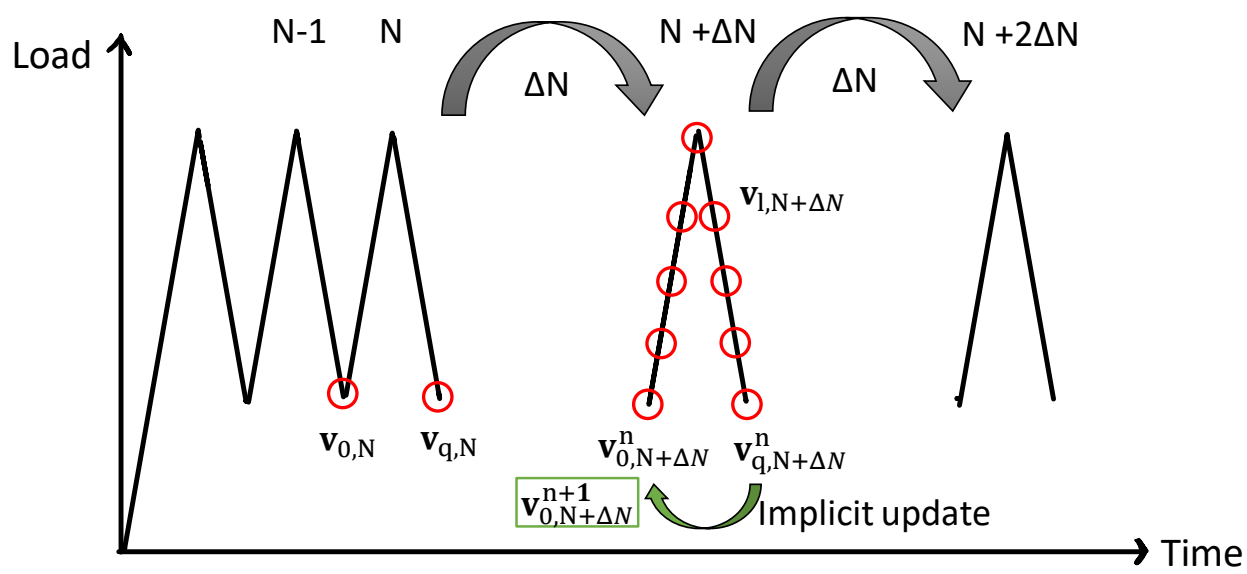

Figure 3: Implicit cycle jump scheme.

\subsubsection{Tangent matrix $\partial \mathbf{v}_{q} /\left.\partial \mathbf{v}_{0}\right|_{N+\Delta N}$}

In this subsection the calculation of derivative $\partial \mathbf{v}_{q} /\left.\partial \mathbf{v}_{0}\right|_{N+\Delta N}$ is presented. For displacement field $\mathbf{u}$ and damage field $d$, we follow [44] and apply the chain rule:

$$
\left.\frac{\partial \mathbf{v}_{q}}{\partial \mathbf{v}_{0}}\right|_{N+\Delta N}=\left.\prod_{m=0}^{q-1} \frac{\partial \mathbf{v}_{q-m}}{\partial \mathbf{v}_{q-m-1}}\right|_{N+\Delta N} .
$$

The change from one time step to another is:

$$
\left.\frac{\partial \mathbf{v}_{l}}{\partial \mathbf{v}_{l-1}}\right|_{N+\Delta N}=\left.\left(\frac{\partial \mathbf{f}}{\partial \mathbf{v}_{l}}\right)^{-1}\left(\frac{\partial \mathbf{f}}{\partial \mathbf{v}_{l-1}}\right)\right|_{N+\Delta N}
$$

where $\mathbf{f}$ is a specific evolution equation for the variable $\mathbf{v}$.

The displacement field is described with $\mathbf{f}^{u}$, the discretization of the weak form of Eq. 25], see [20]:

$$
\mathbf{f}^{u}=\int_{\Omega_{0}} g_{d}\left(\nabla_{0} \mathbf{N}\right)^{T} \mathbf{P} d V-\int_{\Omega_{0}} \mathbf{N}^{T} \mathbf{b}_{0} d V=\mathbf{0},
$$

where $\mathbf{N}$ denotes the standard shape functions and $\mathbf{P}$ the first Piola-Kirchhoff stress, see Eq. 88. The derivatives are calculated as:

$$
\frac{\partial \mathbf{f}^{u}}{\partial \mathbf{u}_{l}}=\mathbf{K}_{l}^{u u}
$$

and

$$
\frac{\partial \mathbf{f}^{u}}{\partial \mathbf{u}_{l-1}}=\mathbf{0}
$$




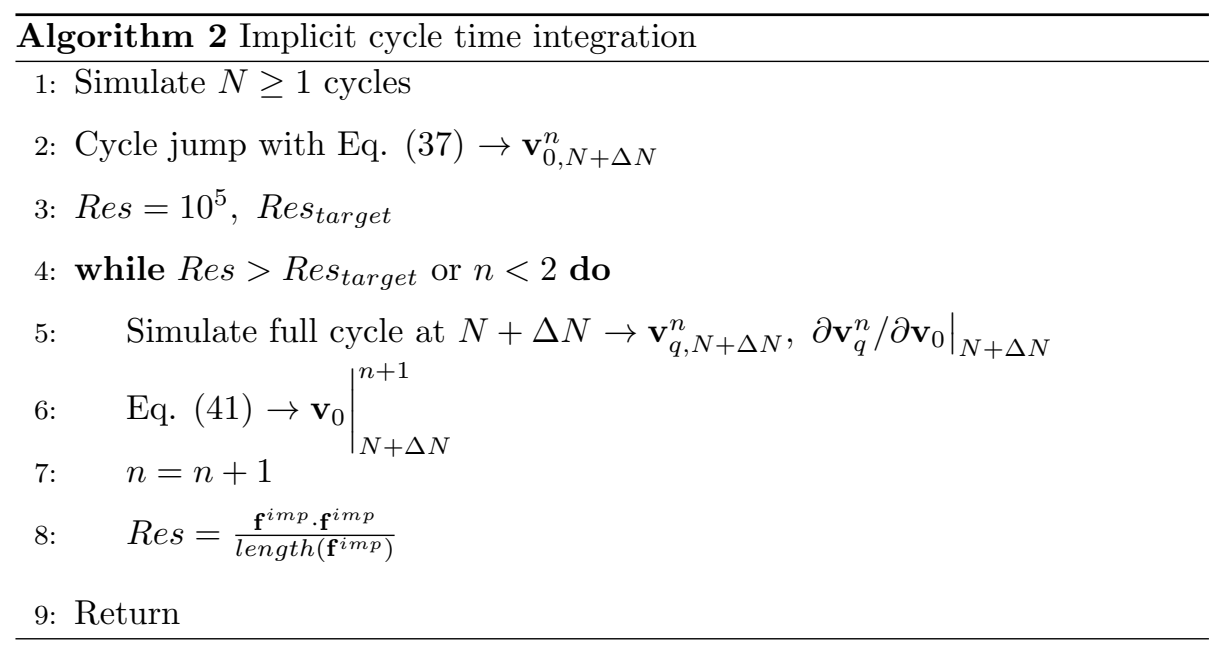

Inserting Eqs. 47 and 48 into Eqs. 45 and 44, we write:

$$
\left.\frac{\partial \mathbf{v}_{q}}{\partial \mathbf{v}_{0}}\right|_{N+\Delta N} ^{\mathbf{u}}=\mathbf{0} \text {. }
$$

Next, we focus on damage $d$. The discretization of the weak form of Eq. 26. leads to:

$$
\begin{aligned}
& \mathbf{f}^{d}=\int_{\Omega_{0}} \frac{2 l_{0}^{2}}{\pi}\left(\nabla_{0} \mathbf{N}\right)^{T}\left(\nabla_{0} d\right) d V \\
&+\int_{\Omega_{0}} \mathbf{N}\left[\frac{\partial g_{d}}{\partial d}\left(\psi^{e l a s}+\zeta_{d} h^{\zeta e}\right) \frac{l_{0}}{G_{c}}+\frac{1}{\pi}(2-2 d)\right] d V, \\
& \frac{\partial \mathbf{f}^{d}}{\partial \mathbf{d}_{l}}=\mathbf{K}_{l}^{d d},
\end{aligned}
$$

and

$$
\frac{\partial \mathbf{f}^{d}}{\partial \mathbf{d}_{l-1}}=\mathbf{0}
$$

Combining Eq. (51) and (52) with Eq. (45) and (44) results in:

$$
\left.\frac{\partial \mathbf{v}_{q}}{\partial \mathbf{v}_{0}}\right|_{N+\Delta N} ^{d}=\mathbf{0 .}
$$

The calculation for the load history $h$ is easier since we can directly write:

$$
h_{q}=h_{0}+s_{l} s_{c} \int \dot{\psi}^{e l a s} d t
$$

and calculate the derivative:

$$
\left.\frac{\partial \mathbf{v}_{q}}{\partial \mathbf{v}_{0}}\right|_{N+\Delta N} ^{h}=\mathbf{I}
$$




\subsubsection{Adaptive time stepping}

An adaptive cycle jump procedure for the implicit scheme is proposed in this subsection. Depending on the non-linearity of the structural response, the jump size is adapted. We simply measure the non-linearity with the number of iterations of the implicit scheme, i.e. $n$.

The number of iterations, $n$, is then compared to $n_{\max }$. We distinguish between three cases:

- $n=n_{\max }$ : The implicit scheme did not converge at all. The jump is repeated with a reduced jump size.

- $n=2$ : The implicit scheme converged fast. The next jump size is increased by factor $N_{\text {scale }}$.

- $\frac{n_{\max }}{2} \leq n<n_{\max }$ : The implicit scheme converged slowly. The next jump size is decreased.

The jump size is at least 3 and maximally $\Delta N_{\max }$. We initialize $\Delta N_{\max }=\infty$, but reduce it to $N_{\max }=\Delta N$ if the scheme did not converge and the jump must be repeated. If the rate of damage before and after the jump is constant, the factor is $N_{\text {scale }}=3$, but it decreases with increasing damage:

$$
N_{\text {scale }}=3 /\left(1+\frac{\sum_{j}^{\text {nodes }}\left(d_{q, N+\Delta N}-d_{0, N+\Delta N}\right)_{j}}{1+\sum_{j}^{\text {nodes }}\left(d_{q, N}-d_{0, N}\right)_{j}}\right) .
$$

The algorithm is summarized in Algo. 3 .

\section{Examples: Explicit and implicit cycle jumps}

In this chapter, we apply the explicit and implicit cycle jump schemes to three different examples and evaluate the speed up. The first simulations use a fixed step size, so that the adaptive method can be evaluated based on the results. For all simulations in this section, we use the material parameters presented in Tabs. 1, 2 and 3,8

\footnotetext{
${ }^{8}$ The presented material parameters are identical to the parameters identified in 1 .
} 


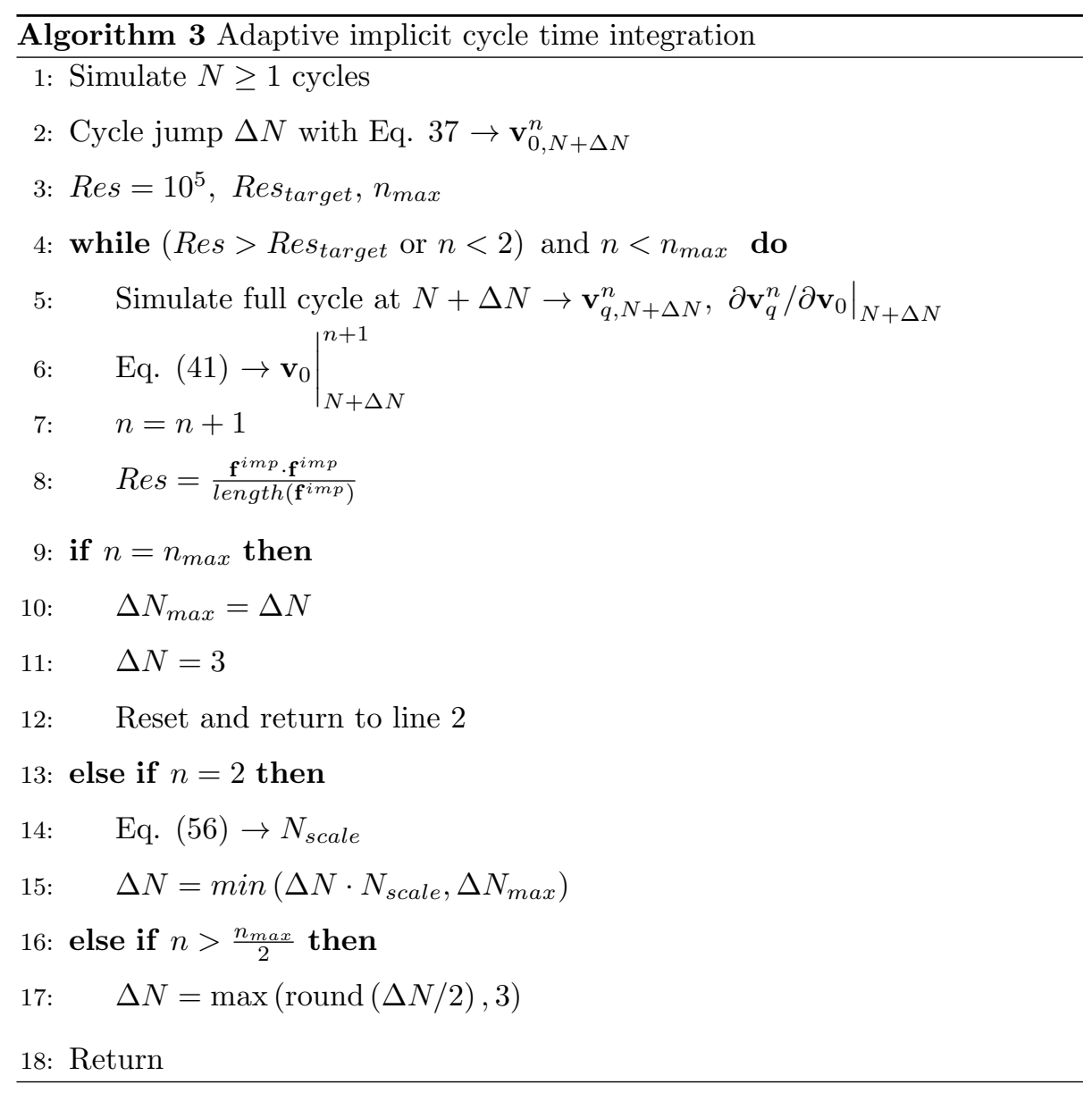


Table 1: Material parameters for the hyperelastic model.

\begin{tabular}{|c|c|c|}
\hline$C_{1}\left[\mathrm{~N} / \mathrm{mm}^{2}\right]$ & $C_{2}\left[\mathrm{~N} / \mathrm{mm}^{2}\right]$ & $C_{3}\left[\mathrm{~N} / \mathrm{mm}^{2}\right]$ \\
\hline 0.8766 & 0.0705 & $1.0763 \cdot 10^{-06}$ \\
\hline
\end{tabular}

Table 2: Phase-field fracture parameters

\begin{tabular}{|c|c|c|}
\hline$G_{c}[\mathrm{~N} / \mathrm{mm}]$ & $l_{0}[\mathrm{~mm}]$ & $a[-]$ \\
\hline 6.0 & 0.25 & 1.88 \\
\hline
\end{tabular}

\subsection{Uniaxial cyclic tension}

At first, we consider uniaxial cyclic tension of a slender bar (length $2.0 \mathrm{~mm}$, width $0.25 \mathrm{~mm}$ and thickness $0.25 \mathrm{~mm}$ ). Unloading takes place until $20 \%$ of the maximum load. We compare in the top of Fig. 4 the number of load cycles to failure for the explicit and implicit schemes with full simulations of all load cycles. Both, the implicit and explicit schemes, use a constant jump $\Delta N=15$. The error from the accelerated calculation is small, but the calculation time (see bottom of Fig. 4 is greatly reduced. Comparing the results for the implicit and explicit scheme, we see that the former is slightly faster. The implicit scheme is faster, since after the jump only one complete cycle has to be simulated, while the explicit scheme requires three cycles for the second-order Taylor expansion (see Eq. (31)). Therefore, we also apply an explicit scheme with a first order Taylor series extrapolation (green dotted line in Fig. 4):

$$
\mathbf{v}_{0, N+\Delta N}=\mathbf{v}_{0, N}+\left.\Delta N \frac{\partial \mathbf{v}_{0}}{\partial N}\right|_{N} .
$$

This scheme also requires the simulation of only one complete cycle after the jump, making it faster than the implicit scheme. But since it is unable to produce converging solutions for more than $N>200$ load cycles, it is not further used.

Critical for the final calculation time is the selected jump $\Delta N$. If $\Delta N$ is too small, the calculation time will not reduce substantially. However, the simula- 
Table 3: Phase-field fatigue parameters

\begin{tabular}{|c|c|c|c|}
\hline$\zeta_{d}\left[\mathrm{~N} / \mathrm{mm}^{2}\right]$ & $\zeta_{e}[-]$ & $\zeta_{t}\left[\mathrm{~N} / \mathrm{mm}^{2}\right]$ & $\zeta_{p}\left[\mathrm{~mm}^{2} / \mathrm{N}\right]$ \\
\hline $1.00 \cdot 10^{-06}$ & 3.0 & 0 & 0 \\
\hline
\end{tabular}

tion may not converge if a too large value is used. We see in Fig. 4 that the error for the constant jump $\Delta N=15$ decreases with the number of load cycles, whereas the time savings relative to the full simulation remains the same.

A study of the influence of jump size $\Delta N$ is presented in Fig. 5. The applied stretch ratio is $\lambda=1.25$. Increasing the jump size, the error increases but the calculation time is reduced. No convergence issues are encountered with the explicit scheme, even with a large jump size of $N=300$. This is mainly due to the simplicity of the uniaxial test case, as we cannot confirm this for the more complex case in Section 4.2. In our opinion, a good compromise between accuracy and speed up is a jump size $\Delta N=75 \approx 10 \% N_{\text {max }, \text { full }}$.

We set, therefore, for the next study the jump to ten percent of the number of load cycles to failure of the full simulations $\left(\Delta N=10 \% N_{\max , \text { full }}\right)$. The results for this jump step size are presented in Fig. 6. While the error is still small, the relative speed up increases. For a stretch ratio $\lambda=1.0625$ the relative error of the results of the full calculation and the implicit scheme is $3.0 \%$, while the implicit scheme is 200 times faster than the full calculation. However, the number of load cycles to failure is normally not known beforehand and we recommend the use of an adaptive cycle jump procedure (see Subsection 4.1.1.

\subsubsection{Uniaxial cyclic tension: Adaptive time stepping}

The performance of the adaptive cycle jump procedure for the implicit scheme is examined in this subsection. We set the initial jump $\Delta N=5, \Delta N_{\max }=\infty$ and $n_{\max }=6$. The results for uniaxial cyclic tension are presented in Fig. 7. All schemes show nearly the same number of load cycles to failure and the calculation time is reduced compared to the implicit scheme with $\Delta N=15$. It 

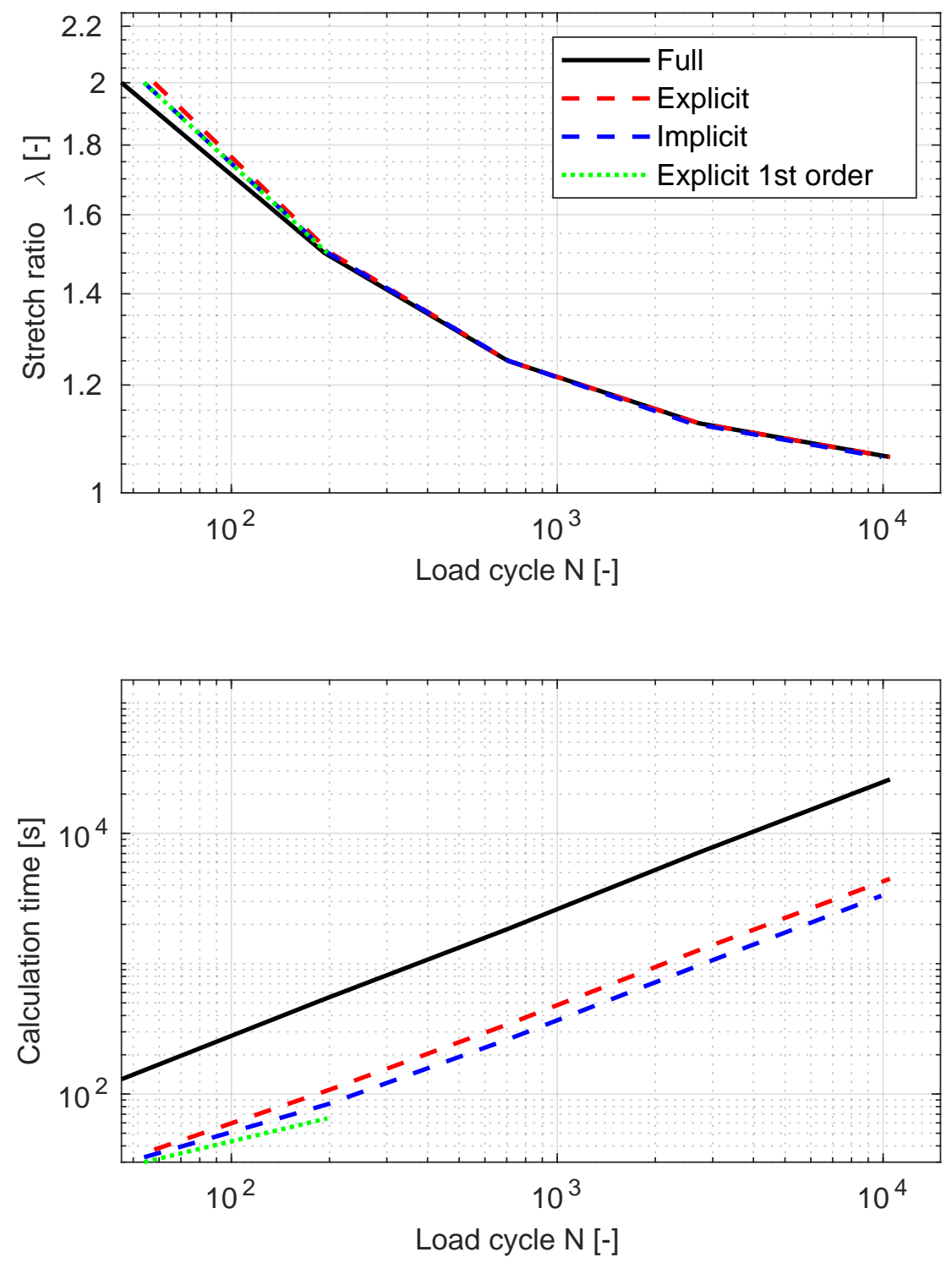

Figure 4: Uniaxial cyclic tension: Stretch ratio versus load cycles to failure (top) and calculation time versus load cycles (bottom). The jump size is fixed to $\Delta N=15$. 

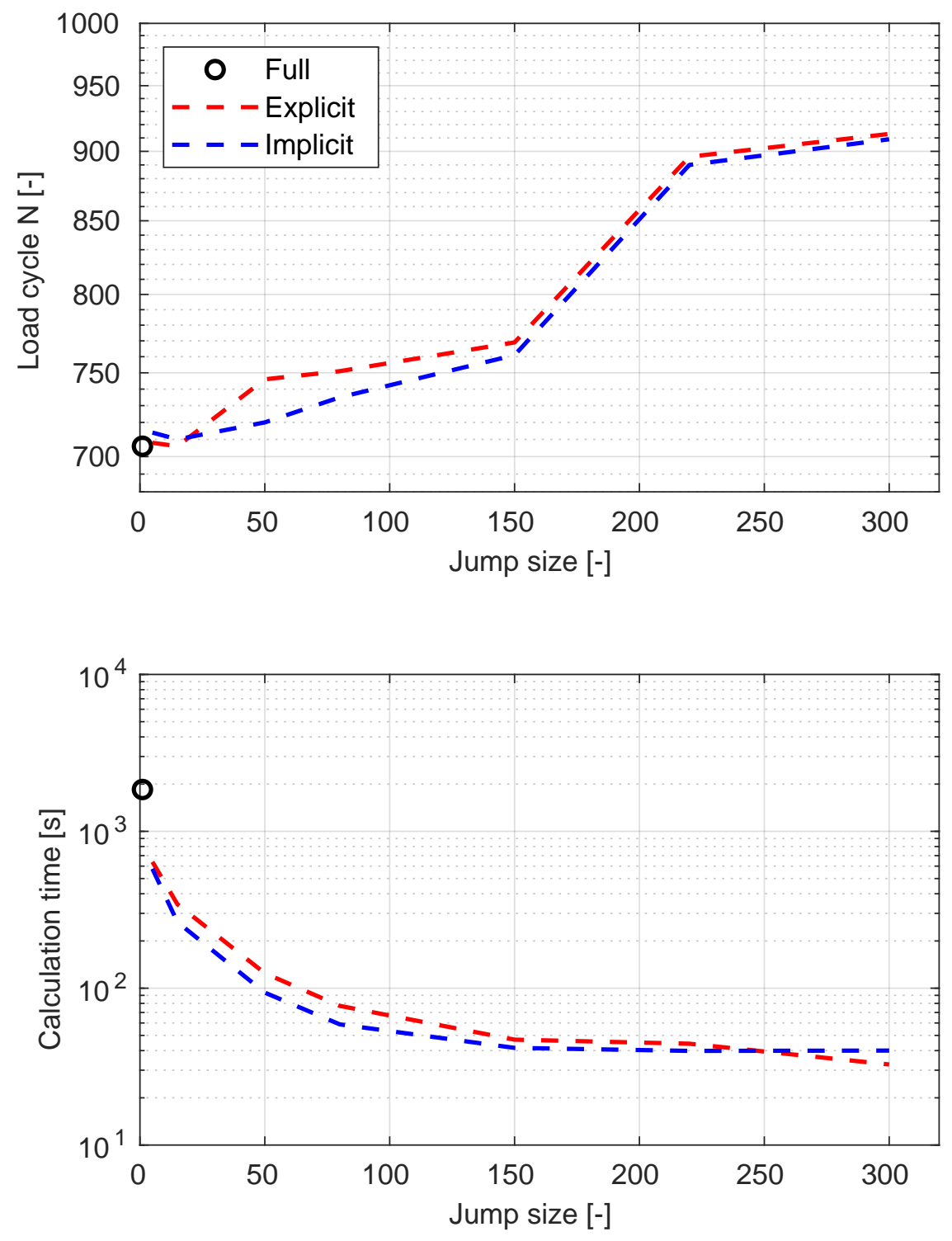

Figure 5: Uniaxial cyclic tension: Load cycles to failure versus jump size (top) and calculation time versus jump size (bottom). The stretch ratio is fixed to $\lambda=1.25$. 

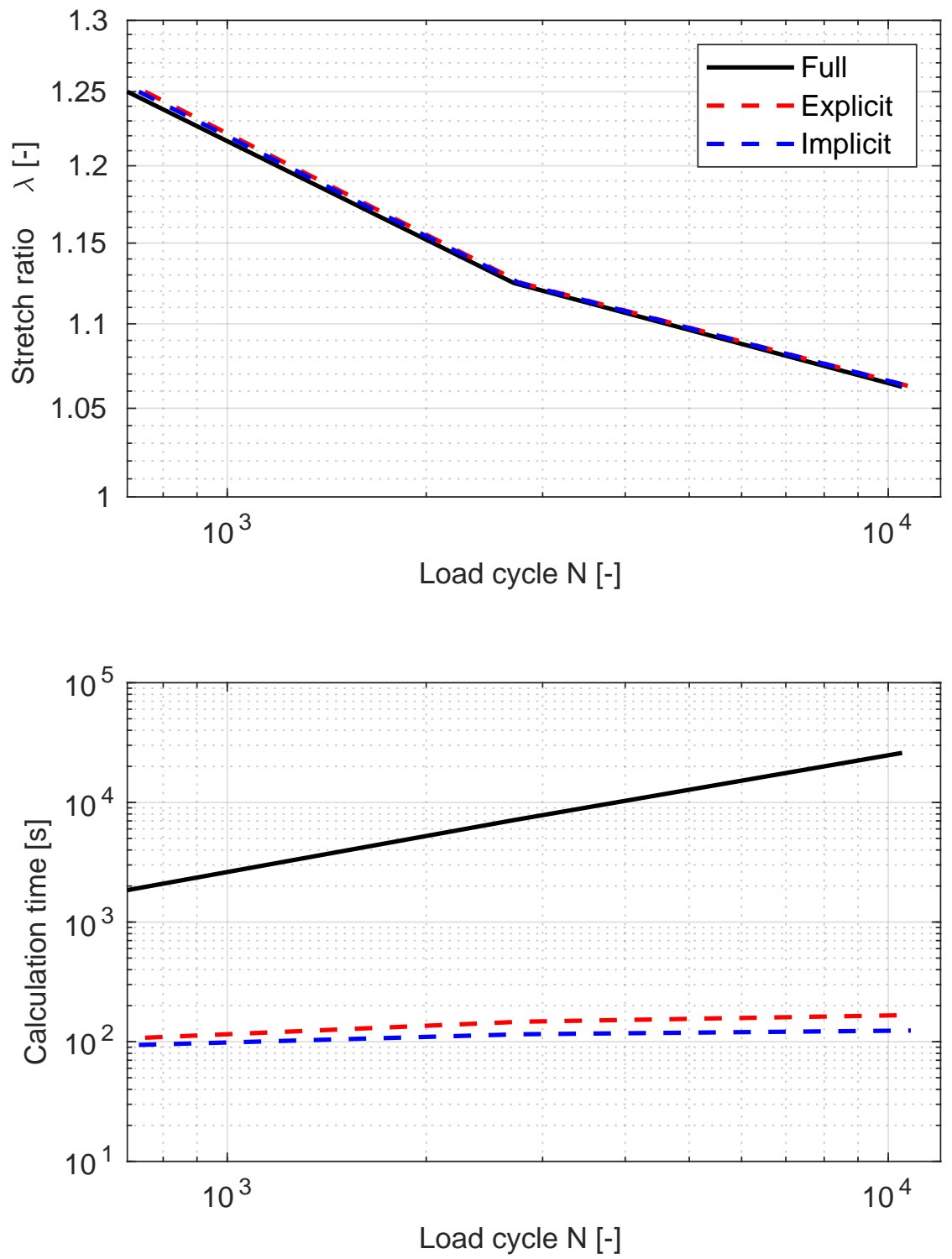

Figure 6: Uniaxial cyclic tension: Stretch ratio versus load cycles to failure (top) and calculation time versus load cycles (bottom). The jump size is fixed to $\Delta N=10 \% N_{\max \text {, full }}$. 
is not as fast as the implicit scheme with $\Delta N=10 \% N_{\max , \text { full }}$, but it does not require $N_{\max , \text { full }}$ to be known beforehand.
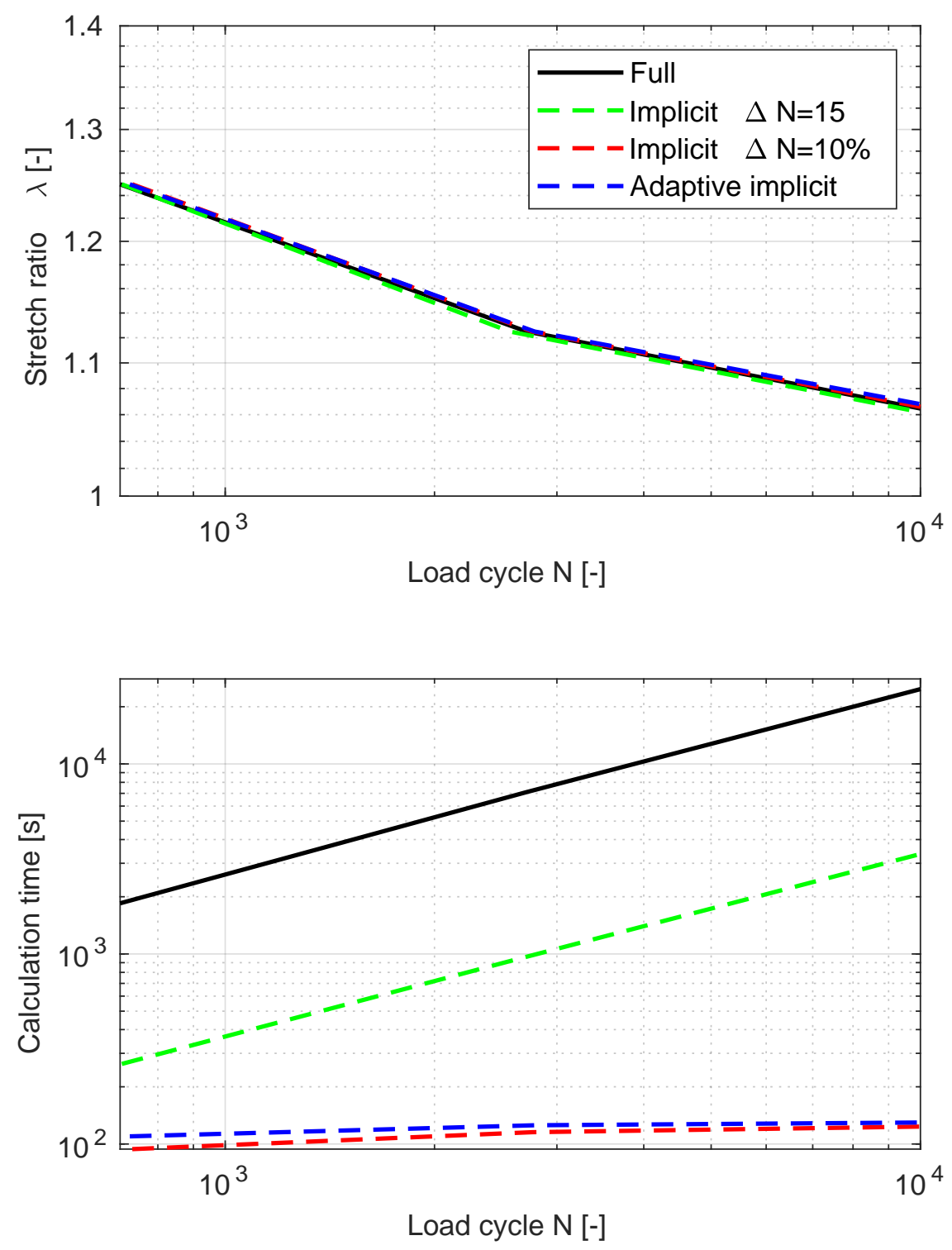

Figure 7: Uniaxial cyclic tension: Stretch ratio versus load cycles to failure (top) and calculation time versus load cycles (bottom). 


\subsection{Single edge notched tensile fatigue test}

The next example considers a specimen with an initial crack. We present in Fig. 8 the dimensions, boundary conditions and the fatigue crack growth for stretch ratio $\lambda=1.5$.

Calculating the load cycles to failure depending on the stretch ratio $\lambda$, the results for the full, explicit, implicit and adaptive schemes are presented in Fig. 9. The jump size is $\Delta N=10$ for all simulations, except for $\lambda=1.5$. Here, the explicit scheme only converges with $\Delta N=2$ and the implicit scheme with $\Delta N=5$. The larger the stretch ratio, the faster history variable grows, as well as the damage variable. This adds more nonlinearity to the system and requires a smaller jump size. The adaptive scheme always starts with $\Delta N=5$.

All schemes reduce the calculation time, while the lifetime is not significantly affected. The implicit scheme is faster than the explicit scheme, while the main advantage of the adaptive scheme is a calculation time which weakly correlates with the number of load cycles to failure.

Interestingly, the calculation time for the adaptive scheme does not increase for an increase in the number of load cycles. A large number of load cycles corresponds to smaller applied stretch (Fig. 9) and hence, the nonlinearity is reduced. This results in turn in large jump sizes and fast calculations. Similar observations are made for the next example (Fig. 13).

A study of the influence of jump size $\Delta N$ is presented in Fig. 10. For all cases, the stretch ratio is $\lambda=1.25$. An increase of the jump size leads to a larger error while the calculation time is reduced. The maximum jump size for the explicit scheme is $\Delta N=15$ and for the implicit $\Delta N=20$. In the previous section, we have concluded that a good compromise between accuracy and speed up is achieved with jump size $\Delta N=10 \% N_{\max , \text { full }}$. This jump size would be too large for the current case and highlights that the selection should not only depend on the load level (Figs. 5 and 10 both present results for $\lambda=1.25$ ), but also on the test case.

Additionally, we compare in Fig. 11 the final crack path for the full, direct numerical simulations and all acceleration schemes. Due to the different jump 
sizes and load cycles to failure, the plots are at different discrete load cycles.

For stretch ratio $\lambda=1.125-1.5$, only a small deviation between the results of the different schemes and the direct numerical simulation can be observed.

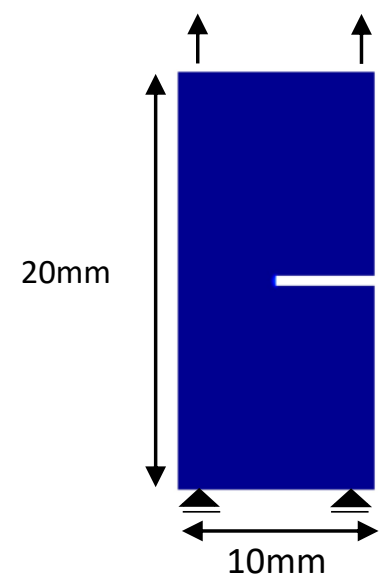

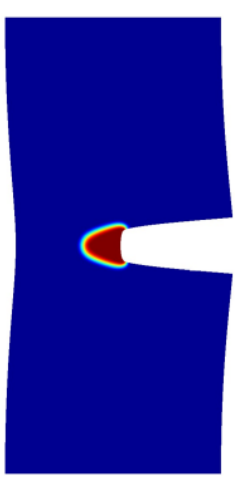

$\mathrm{N}=27$

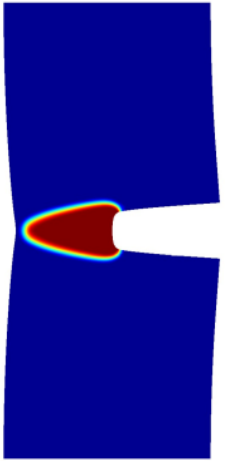

$\mathrm{N}=66$

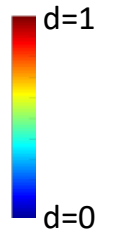

Figure 8: Single edge notched tensile fatigue test: Dimensions of the sample, boundary condition and fatigue crack growth for stretch ratio $\lambda=1.5$ predicted by the direct numerical simulation. 

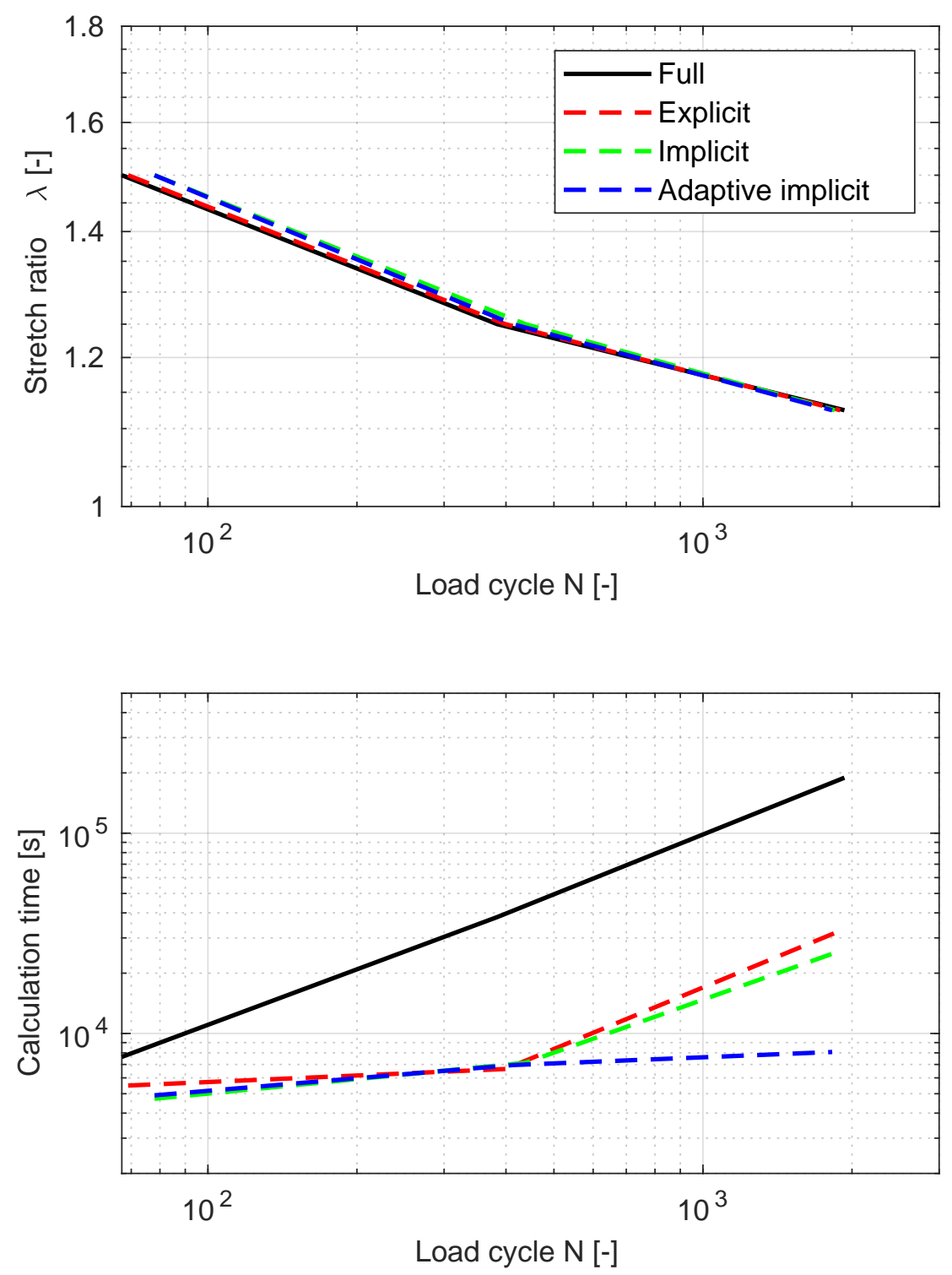

Figure 9: Single edge notched tensile fatigue test: Stretch ratio versus load cycles to failure (top) and calculation time versus load cycles (bottom). 

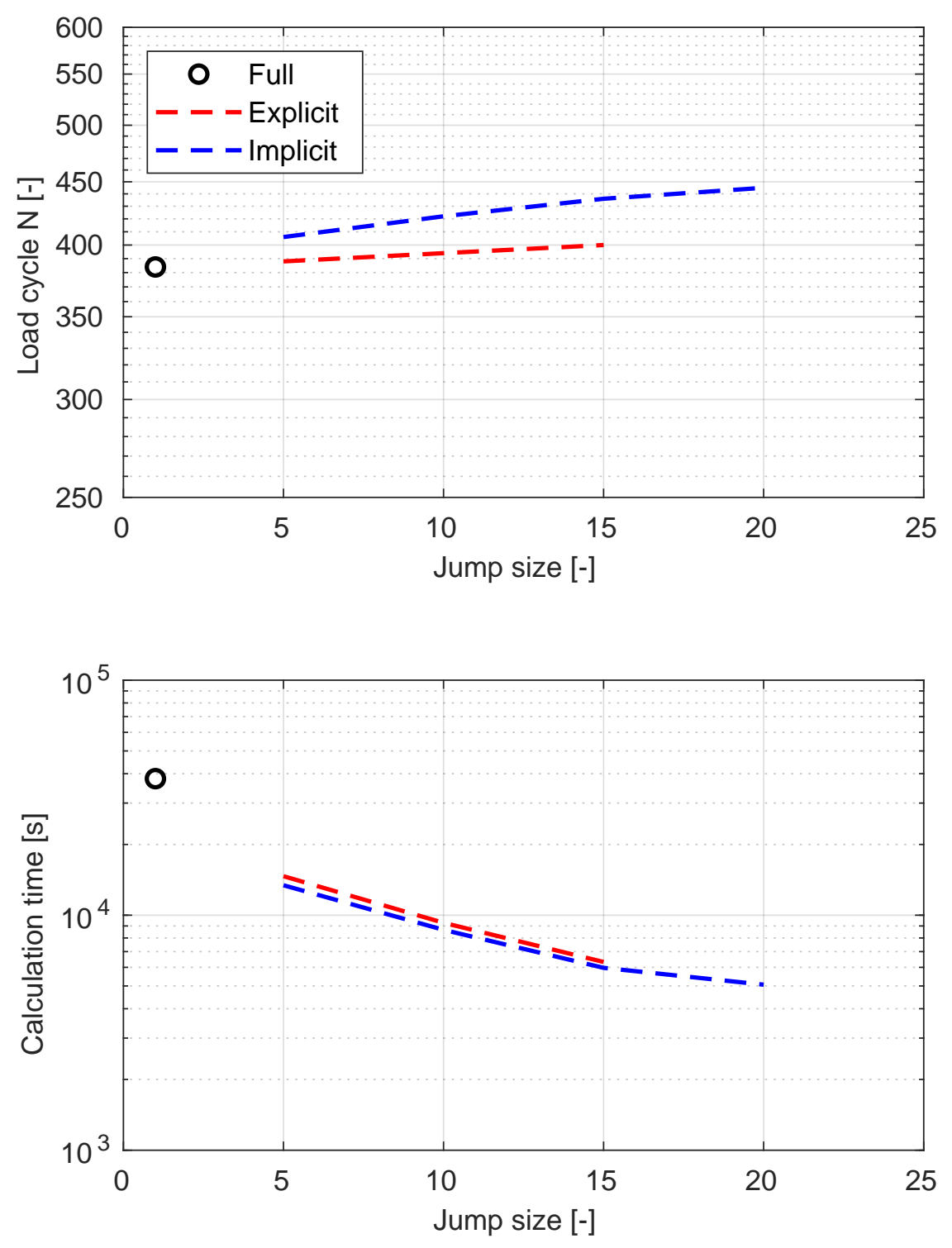

Figure 10: Single edge notched tensile fatigue test: Load cycles to failure versus jump size (top) and calculation time versus jump size (bottom). The stretch ratio is fixed to $\lambda=1.25$. 


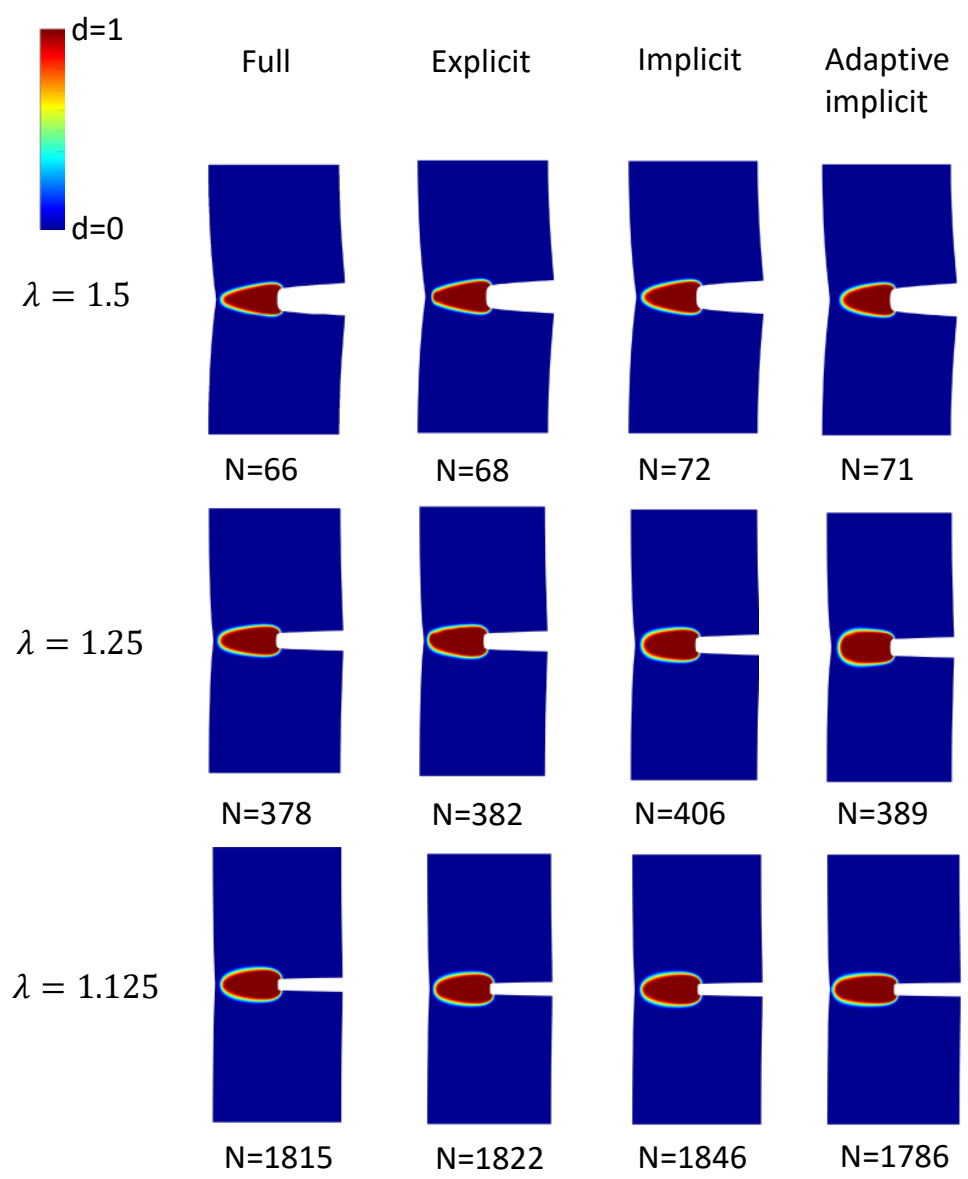

Figure 11: Single edge notched tensile fatigue test: Final crack paths for stretch ratio $\lambda=$ $1.125-1.5$. 


\subsection{Bending beam test}

The next example considers a more complex geometry and includes regions with tensile and compressive stresses. The geometry and boundary conditions are presented in Fig. 12. We calculate the load cycles to failure depending on the displacement boundary condition for the full cycle by cycle simulation and the implicit scheme with adaptive cycle jump size.

For a prescribed displacement $u_{b}=12 \mathrm{~mm}$ at the right end of the beam, the crack nucleates after 295 load cycles and the part fails after 315 cycles (see Fig. 12. The crack initiates at the top, the region of the maximum tensile stress. While the implicit scheme shows a calculation time, which does not correlate the number of load cycles, the computing time for the full cycle by cycle simulation increases significantly (see Fig. 13. A full cycle by cycle calculation is computationally too costly for $u_{b} \leq 6 \mathrm{~mm}$.

In Fig. 14, we compare the final crack geometries predicted by the direct numerical simulations and the adaptive implicit scheme for a prescribed displacement $u_{b}=6-12 \mathrm{~mm}$. Again, for all loads, only a small deviation can be observed.

In summary, we can state that both the explicit and implicit schemes reduce the calculation time. The implicit scheme is slightly faster than the explicit scheme that is based on second-order Taylor series. Explicit procedures based on first-order Taylor series are not recommended as convergence is not always guaranteed. The reduction of the computation time, but also the accuracy of the results compared to those of the direct numerical simulation mainly depend on the selected jump size.

On the one hand, if the jump size is too small, the speed up is only marginal. On the other hand, if the jump size is too large, the simulation might not converge. To select an appropriate jump size, not only the specific case, but also the expected lifetime must be considered. Slightly larger jump sizes can be used for the implicit scheme than for the explicit scheme.

The presented adaptive scheme automatically adjusts the jump size depending on the required number of iterations. As a result, computing times that are almost independent of the number of load cycles are achieved. Hence, we use the adaptive implicit scheme in the remainder of this study. 


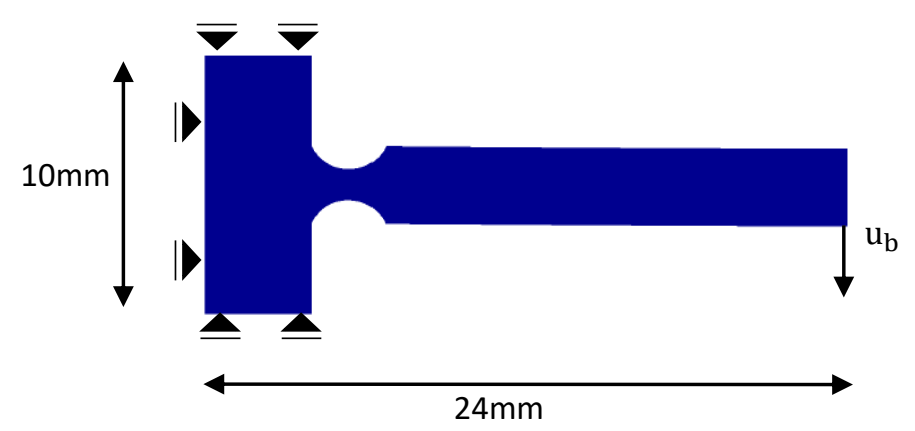

$\mathrm{N}=209$

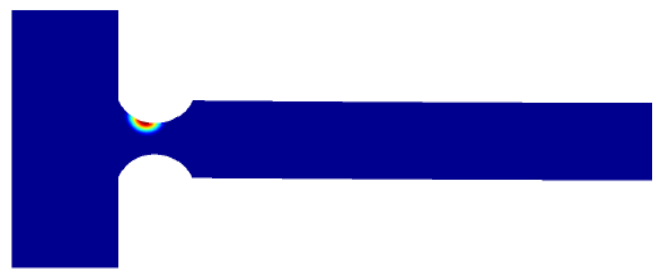

$N=298$
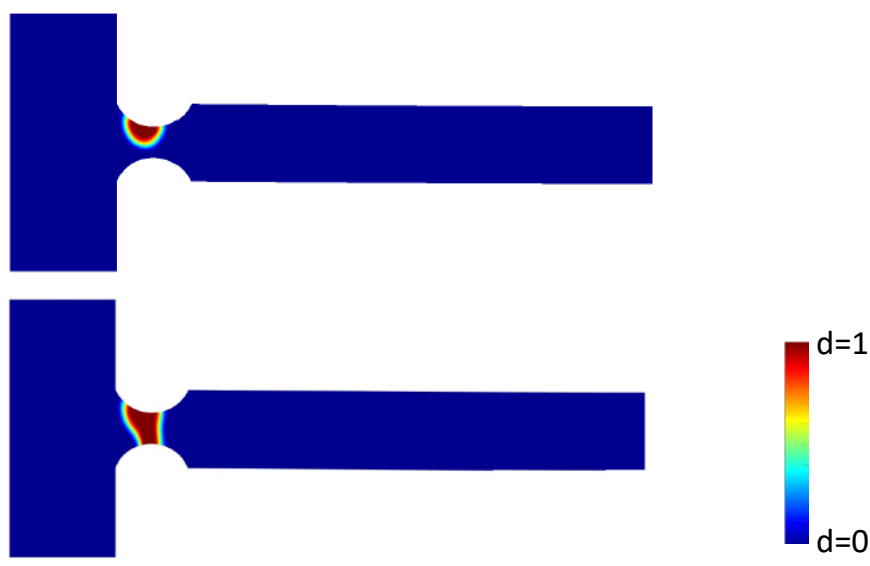

Figure 12: Bending beam test: Dimensions of the sample, boundary condition and fatigue crack growth for displacement $u_{b}=12 \mathrm{~mm}$ predicted by the adaptive scheme. 

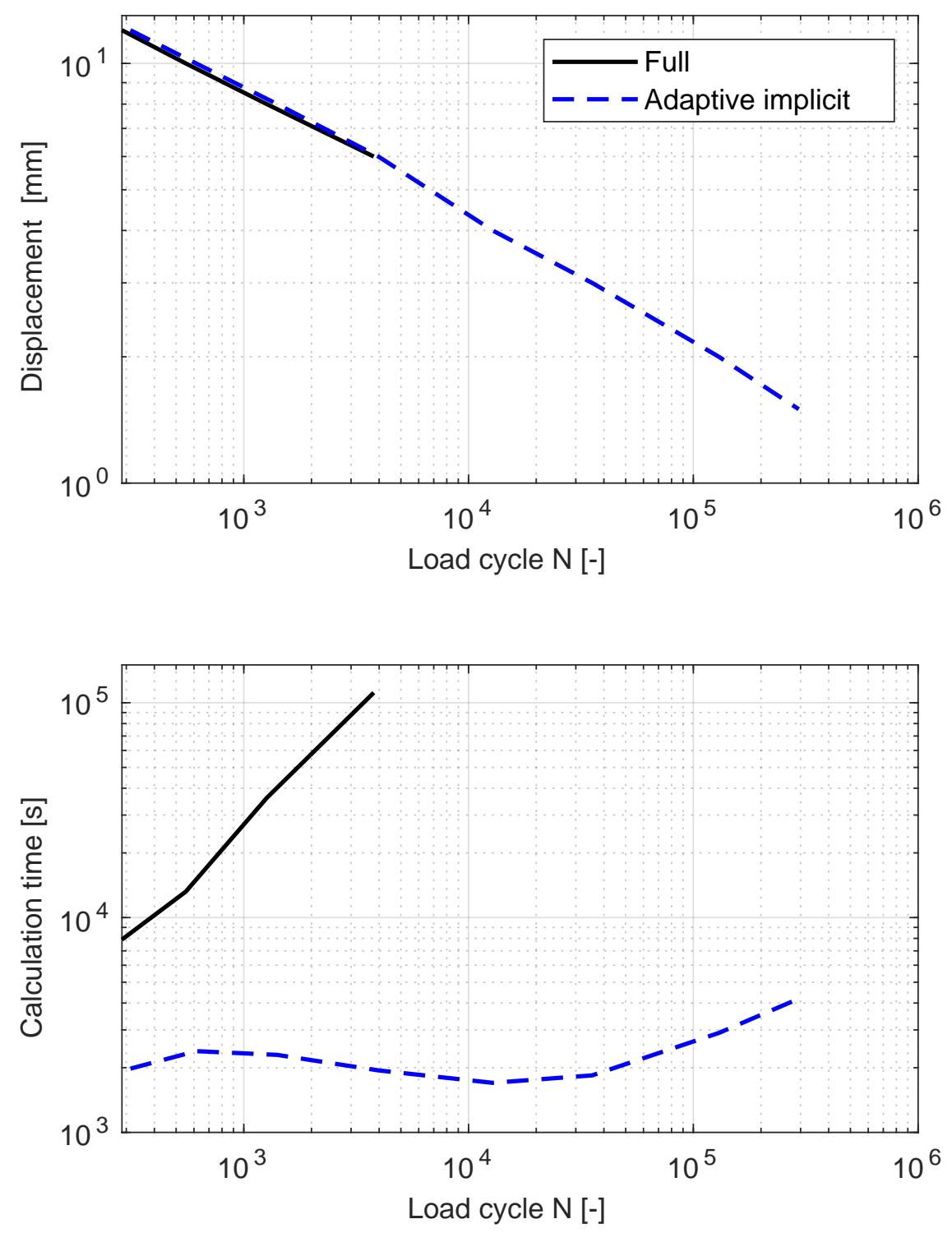

Figure 13: Bending beam test: Displacement versus load cycles to failure (top) and calculation time versus load cycles (bottom). 


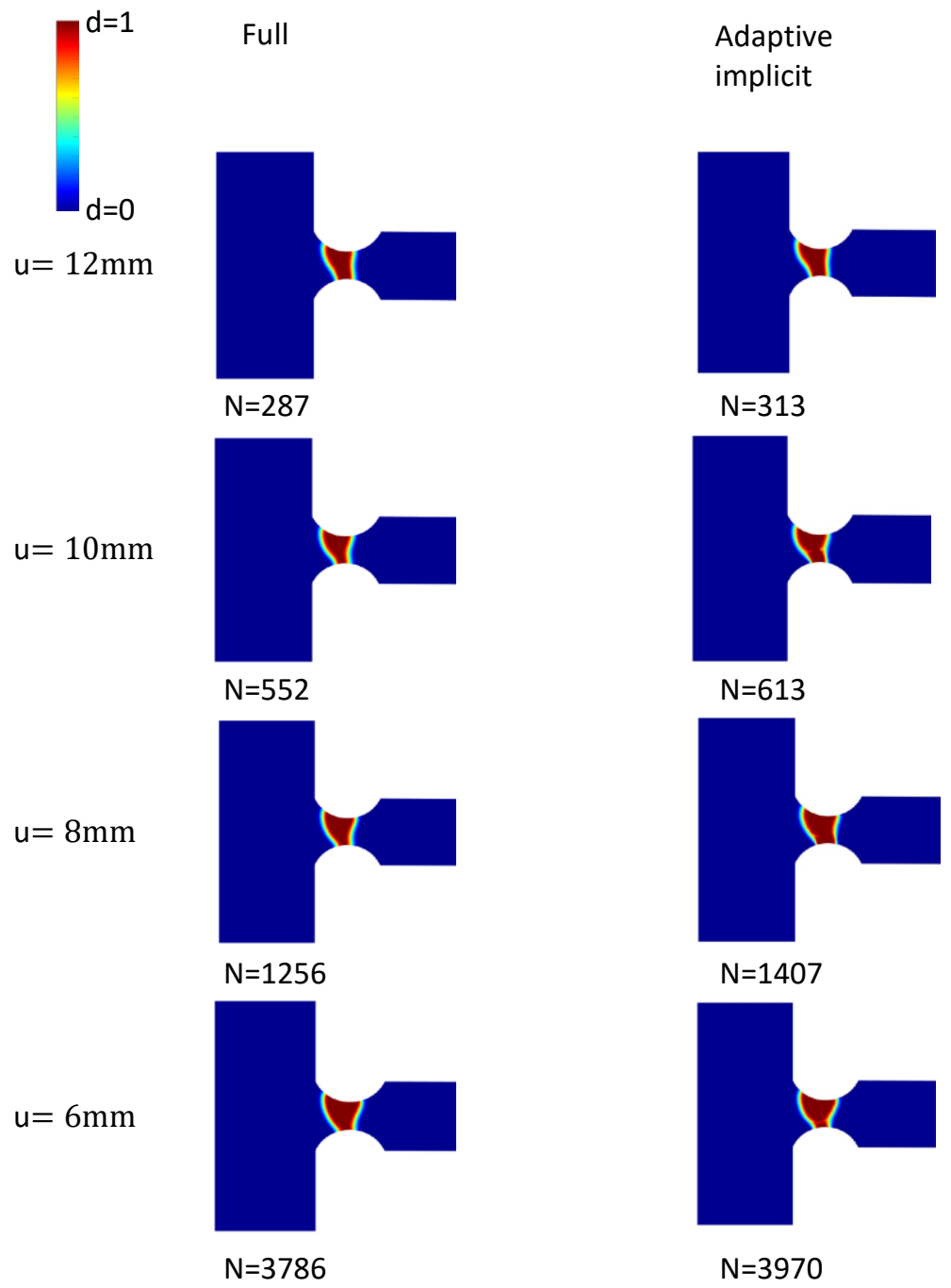

Figure 14: Bending beam test: Fatigue cracks for displacement $u_{b}=6-12 \mathrm{~mm}$. 


\section{Results: Experimental validation}

We experimentally validate the model in this chapter. First, we explain the material parameter identification, followed by experimental and numerical results for fatigue crack nucleation and crack propagation. Plane stress conditions occur in all experiments and are also incorporated in all simulations.

\subsection{Material parameters}

An EPDM rubber is used for all experiments at a constant temperature of $20^{\circ} \mathrm{C}$. Since the focus of this work is on fatigue failure, the hyperelastic material parameters are fitted to the reloaded uniaxial response between stretch ratio $\lambda=1.5-2.9$. Note that the initial material response is much stiffer. The parameters are presented in Tab. 4 and the fit in Fig. 15.

Table 4: Identified hyperelastic material parameters.

\begin{tabular}{|c|c|c|}
\hline$C_{1}\left[\mathrm{~N} / \mathrm{mm}^{2}\right]$ & $C_{2}\left[\mathrm{~N} / \mathrm{mm}^{2}\right]$ & $C_{3}\left[\mathrm{~N} / \mathrm{mm}^{2}\right]$ \\
\hline 0.2807 & 0.01045 & $1.0213 \cdot 10^{-06}$ \\
\hline
\end{tabular}

The phase-field fracture parameters are shown in Tab. 5. Parameters $l_{0}=0.25$ and $a=1.88$ are taken from our previous work ([1]), while fracture toughness $G_{c}=2.25 \mathrm{~N} / \mathrm{mm}$ is adapted to the results for monotonic loading (see load cycle $N=1$ in Figs. 16 and 20p. The adjustment of $G_{c}$ is necessary since we neglect rate-dependent effects in this work (see Tab. 44. We want to point out that $l_{0}=0.25 \mathrm{~mm}$ was fitted to local strain measurements close to crack tip ([1]). The fatigue material parameters (Tab. 6) are identified by minimizing a leastsquares objective function with the experimental load cycles to failure of uniaxial tensile tests (Section 5.2) and single edge notched tensile fatigue test (Section 5.3 with a genetic algorithm. Hence, the results of pure-shear crack growth in Section 5.4 are true predictions without further calibration of the parameters. 
Table 5: Identified phase-field fracture material parameters.

\begin{tabular}{|c|c|c|}
\hline$G_{c}[\mathrm{~N} / \mathrm{mm}]$ & $l_{0}[\mathrm{~mm}]$ & $a[-]$ \\
\hline 2.25 & 0.25 & 1.88 \\
\hline
\end{tabular}

Table 6: Identified phase-field fatigue material parameters.

\begin{tabular}{|c|c|c|c|}
\hline$\zeta_{d}\left[\mathrm{~N} / \mathrm{mm}^{2}\right]$ & $\zeta_{e}[-]$ & $\zeta_{t}\left[\mathrm{~N} / \mathrm{mm}^{2}\right]$ & $\zeta_{p}\left[\mathrm{~mm}^{2} / \mathrm{N}\right]$ \\
\hline $4.01 \cdot 10^{-06}$ & 1.9 & 1.6 & 25 \\
\hline
\end{tabular}

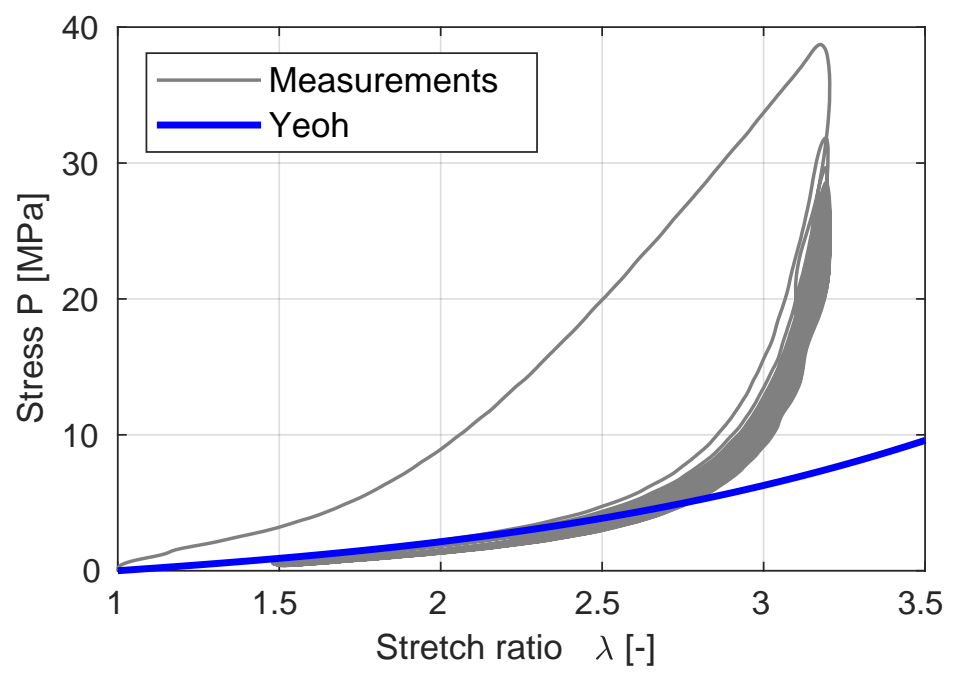

Figure 15: Uniaxial tensile test: Stress versus stretch-ratio. We compare the material model to the experimental observation.

\subsection{Uniaxial tensile test}

A dumbbell specimen according to ISO 37 is subjected to a cyclic load. We measure the number of load cycles to failure depending on the applied maximum stretch. The minimum stretch ratio for all load levels is $\lambda_{\min }=1.5$. Fig. 16 shows the experimental and numerical results. The prediction of the lifetime agrees well with the experimental median value. 


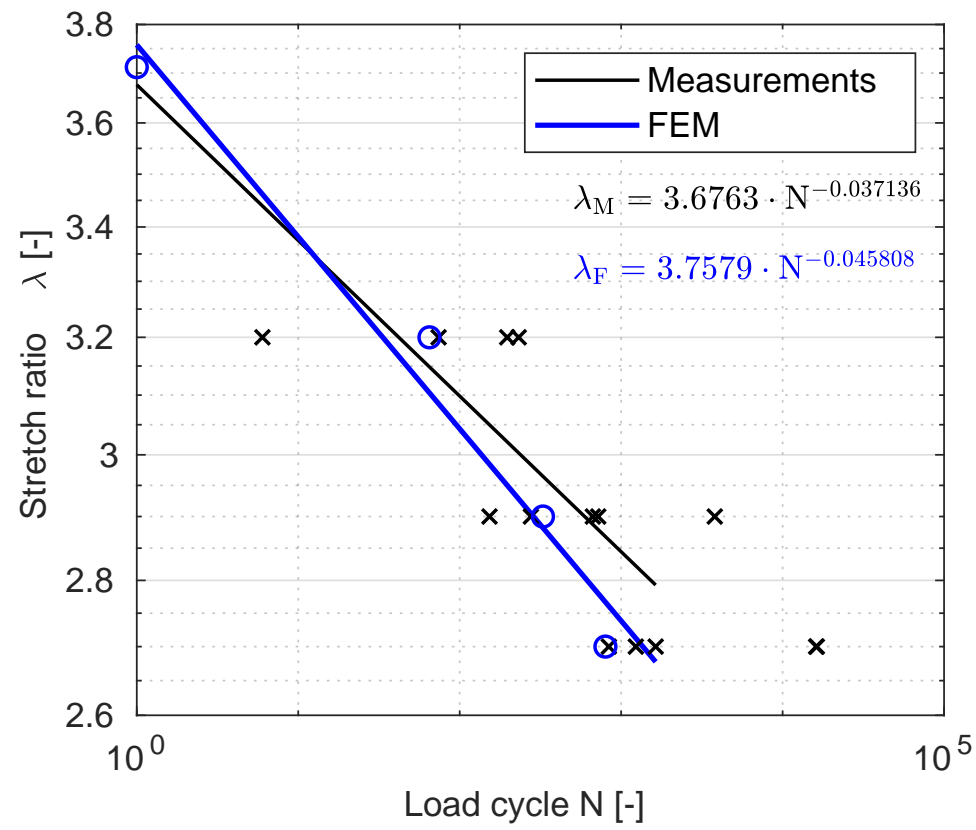

Figure 16: Fatigue uniaxial tensile test: Stretch ratio versus load cycle to failure. The lines present the least-squares trends of the experimental results (black crosses) and the simulations (blue circles).

We note that, the experimental results show a wide scattering. A reason for this could be the random dispersion of large agglomerates. It is known, see e.g. [58] or [71, that fatigue failure of rubber is dependent on the size and distribution of initial flaws. Samples with early failure were characterized with one or more large flaws on the fracture surface. Cracks originate at this location and grow until final rupture (see Fig. 17). 
a)

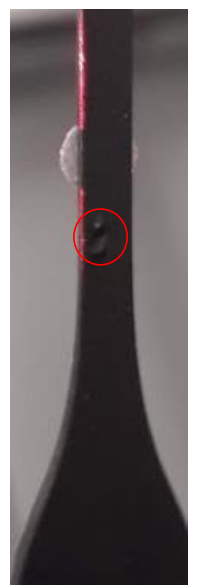

b)

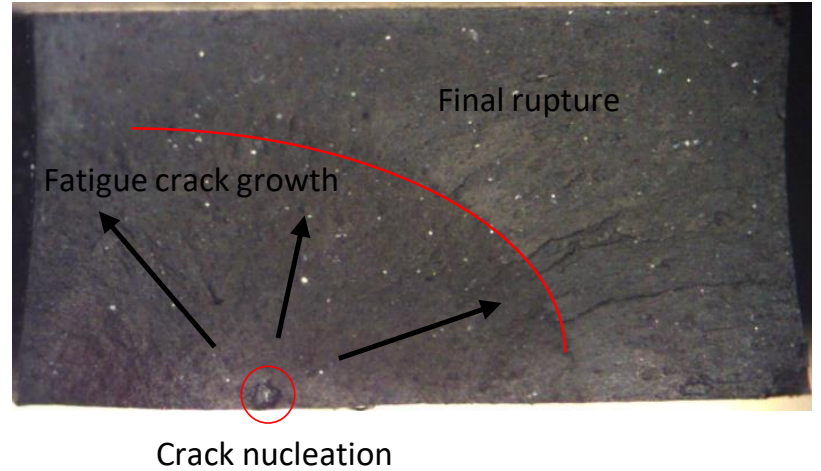

\section{Crack nucleation}

Figure 17: Fatigue uniaxial tensile test: Crack nucleation (a) and final fracture surface (b). The crack initially formed at the largest inclusion. 


\subsection{Single edge notched tensile fatigue test}

Next, we investigate fatigue crack growth for specimens according to ISO 349 The dimensions, boundary conditions and the numerically predicted crack growth for monotonic loading are presented in Fig. 18. In Fig. 19, an experimental ruptured sample is documented. The numerically predicted crack path is identical to its experimental counterpart. Furthermore, we compare the numerically predicted and experimentally measured number of load cycles to failure (Fig. 20. The prediction of our model agrees well with the experimental results.

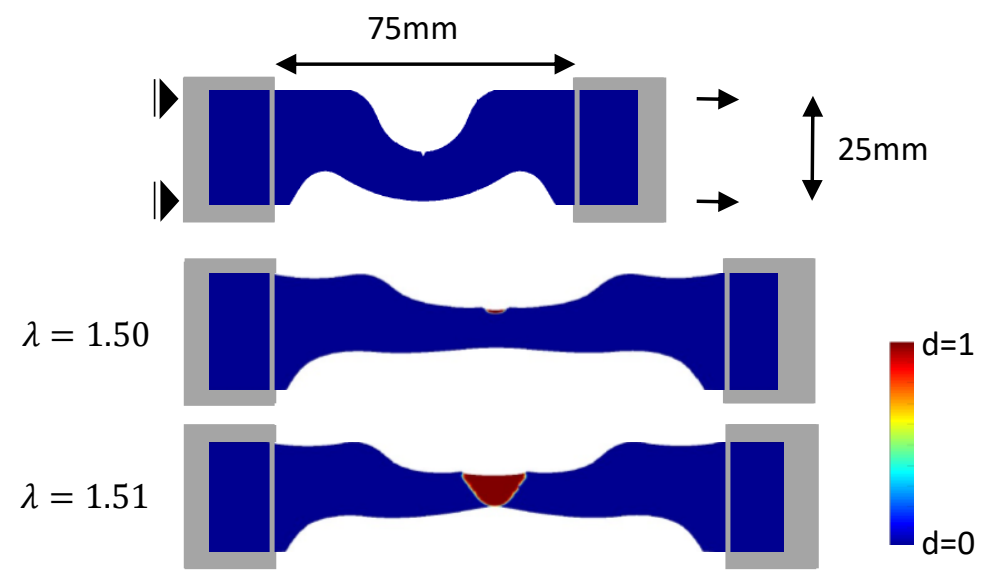

Figure 18: SENT according to ISO34-1, type C: Specimen dimensions, boundary condition and numerically predicted damage field for monotonic loading.

\footnotetext{
${ }^{9}$ The experimental results are taken from [1. We observe a poorer match between the model and the experiments, since we also consider uniaxial tensile test data in the parameter identification.
} 
a)

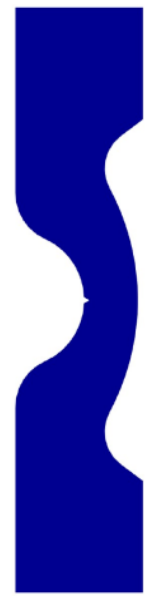

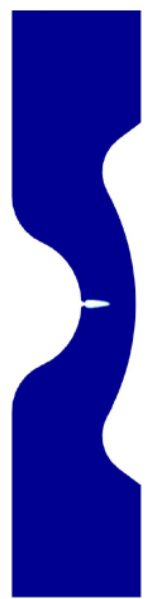

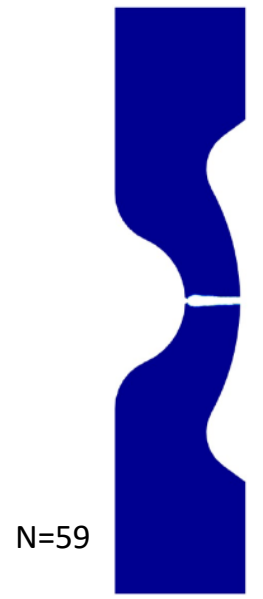

b)

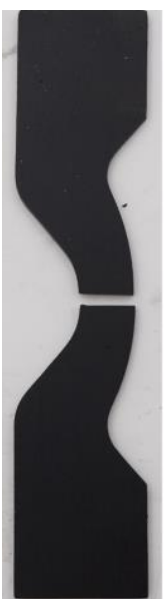

Figure 19: SENT according to ISO34-1, type C: a) Numerically predicted fatigue crack growth for stretch ratio $\lambda=1.4$. Elements with $d>0.95$ are not plotted. b) Experimentally observed crack geometry. 


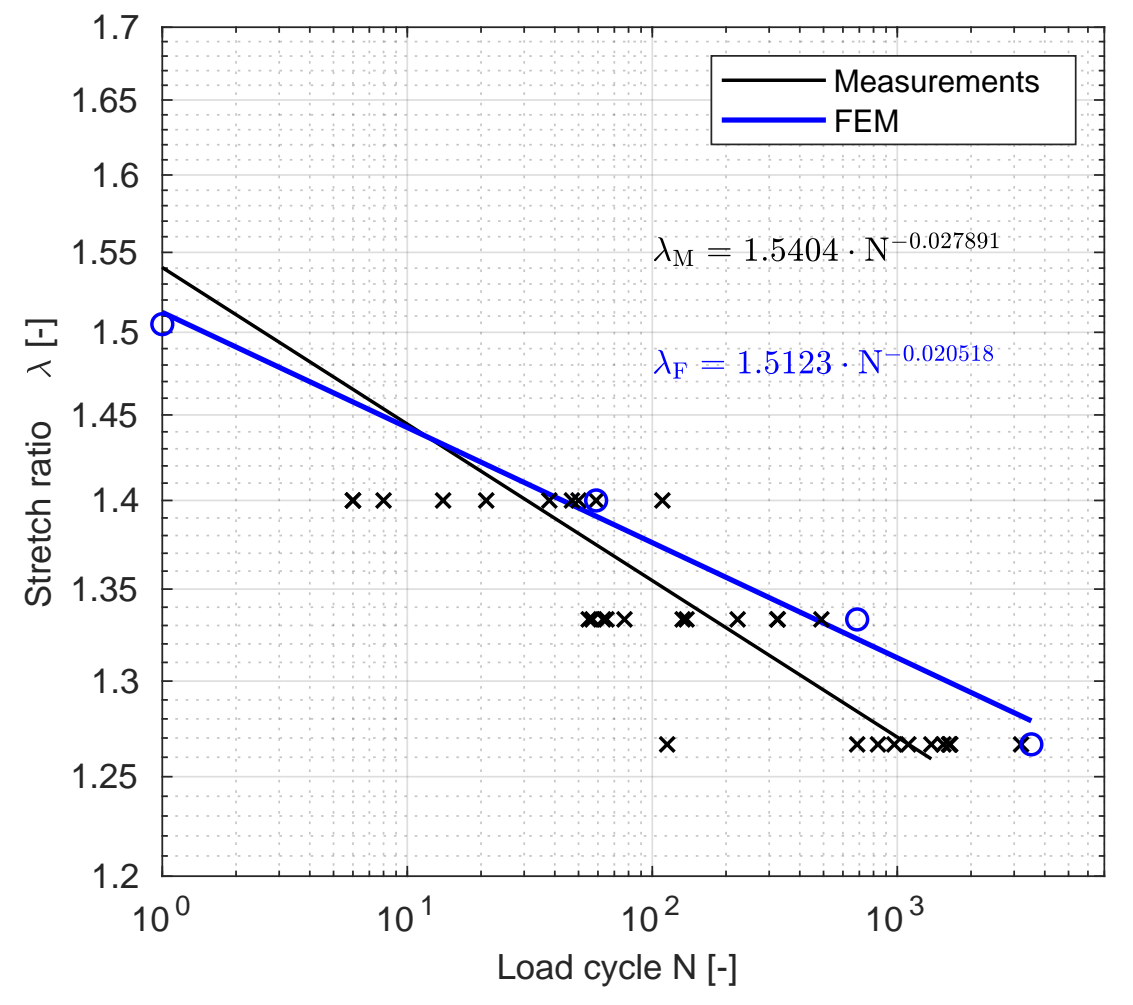

Figure 20: Fatigue SENT according to ISO34-1, type C: Stretch ratio versus load cycle to failure. The lines present the least-squares trends of the experimental results (black crosses) and the simulations (blue circles). 


\subsection{Pure shear fatigue crack growth}

Finally, we validate the model by measuring the fatigue crack growth rate in a pure shear setting according to ISO 27727. The pure shear specimen is characterized by a large width relative to its height and is presented in Fig. 21 . Applying a cyclic load, the crack length can be measured as a function of the number of load cycles (Fig. 22). Two samples were tested and the average crack growth rate as a function of the tearing energy $G=z_{o} \psi^{\text {elas }}$ is presented in Fig. 23. Comparing the experimental results to the model predictions, we see a good correlation. The damage field (Fig. 21) appears large, but is the consequence from a length scale $l_{0}=0.25 \mathrm{~mm}$ experimentally obtained from local strain measurements and a small sample height $\left(z_{0}=2 \mathrm{~mm}\right)$. The kinking of the crack in the tests also indicates a larger process zone at the crack tip (Fig. 22).

a)

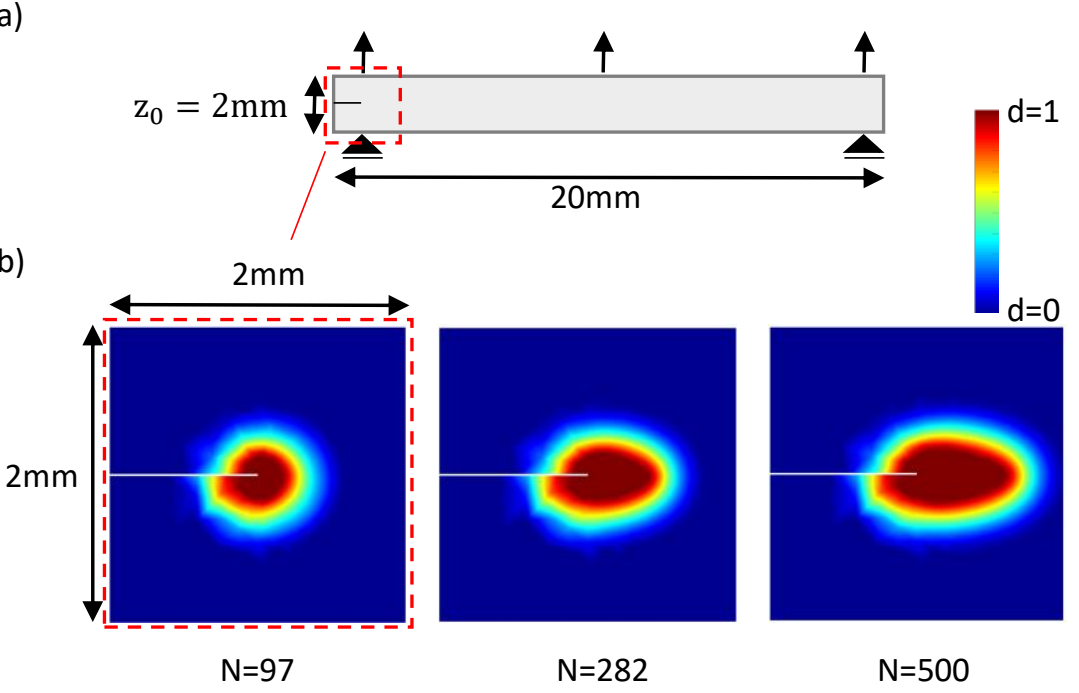

Figure 21: Pure shear fatigue crack growth: a) Dimensions and boundary conditions. The minimal force in a load cycle is zero. b) Damage propagation at the crack tip. 

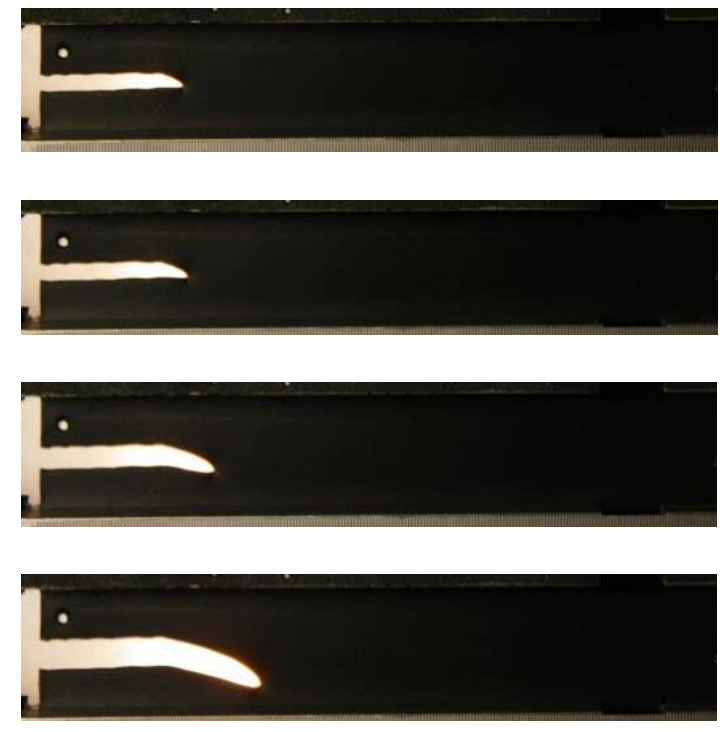

Figure 22: Pure shear fatigue crack growth: Experimentally observed crack geometry (from top to bottom). 


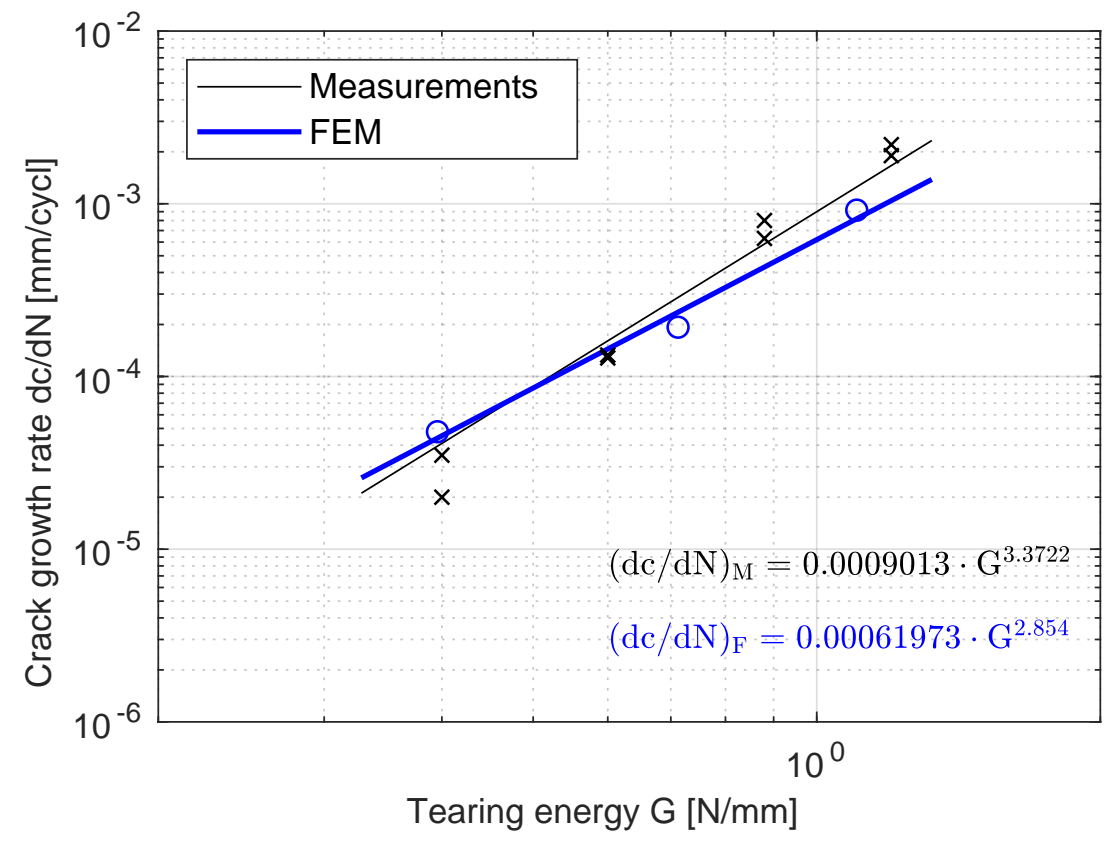

Figure 23: Pure shear fatigue crack growth: Crack growth rate versus the tearing energy. The lines present the least-squares trends of the experimental results (black crosses) and the simulations (blue circles). 


\section{Concluding remarks}

In the context of phase-field damage models, an extension to predict fatigue damage is achieved by introducing a fatigue damage source in the balance of mechanical energy. To accelerate fatigue simulations, explicit and implicit cycle jump schemes are presented. Both schemes reduce the calculation time significantly, while the accuracy remains largely unaffected. As the size of the cycle jump step governs the speed up of the simulations, but may also be responsible for the failure of a simulation, an adaptive cycle jump scheme for the implicit acceleration framework is proposed based on the number of iterations. The maximum acceleration achieved by the implicit scheme was $99.5 \%$.

Thanks to extensive measurements, all material parameters are identified from experiments. The adaptive implicit scheme decreases the computation times so much that we were even able to identify the fatigue material parameters - with uniaxial fatigue tensile tests on dumbbell specimens (ISO 37) and fatigue crack growth experiments according to ISO 34. While the lifetime of the dumbbell specimens is underestimated, the lifetime of specimens according to ISO 34 is slightly overestimated. We validate the model and identified material parameters with fatigue crack growth measurements according to ISO 27727.

These examples suggest that the fatigue phase-field damage model is able to describe the three stages of (fatigue) failure: crack initiation, crack propagation and final fracture. Future work will focus on a three-dimensional implementation so that the influence of multiaxial loading can more accurately be investigated. Furthermore, high-cycle fatigue experiments would experimentally validate the model for more than $10^{6}$ load cycles. 


\section{Appendix A. Parametric study: Fatigue parameters $\zeta_{d}$ and $\zeta_{e}$}

Considering uniaxial cyclic tension and measuring the cycles to failure, we present in Fig. A.24 so-called Woehler lines. We use the hyperelastic and phasefield fracture material parameters from Tabs. 4 and 5 . The fatigue phase-field parameters $\zeta_{d}$ and $\zeta_{e}$ are varied as shown in Fig. A.24 $\left(\zeta_{p}=0\right.$ and $\left.\zeta_{t}=0\right)$.

The multiplier (fatigue material parameter $\zeta_{d}$ ) yields a shift of the response, whilst the exponent (fatigue material parameter $\zeta_{e}$ ) also yields a slope difference. We conclude from the results that Eq. 22 provides a suitable framework to describe the experimental data. 

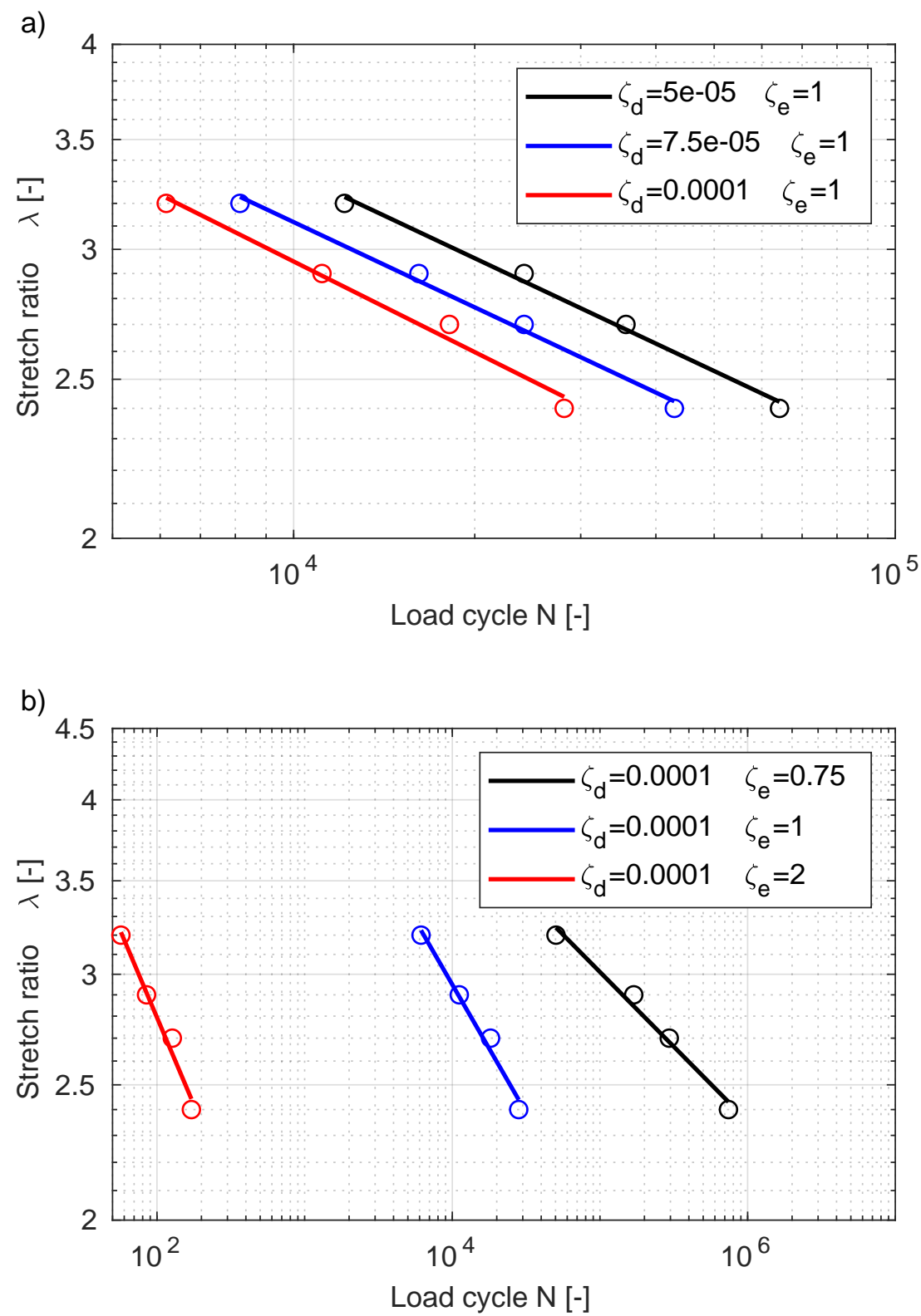

Figure A.24: Uniaxial cyclic tension: Stretch ratio versus number of load cycles to failure for different values of multiplier $\zeta_{d}$ (top) and exponent $\zeta_{e}$ (bottom). 


\section{Appendix B. New load history $h$}

To demonstrate the characteristic of the new load history $h$ we consider two test cases: Uniaxial tension-compression and equibiaxial tension. We use the material parameters presented in Section 4 (Tabs. 1, 2 and 3 ) with $\zeta_{t}=1.6$ and $\zeta_{p}=50$. For the first case of uniaxial tension-compression (see Fig. B.25, we show that the load history does not grow for compressive stresses. Applying equibiaxial tension (Fig. B.26), we see that after reaching the threshold $\zeta_{t}$ the load history grows faster.

This effect is shown in more detail in Fig. B.27, by first varying $\zeta_{p}$ and then $\zeta_{t}$. Fatigue material parameter $\zeta_{p}$ yields a shift of the slope, whilst fatigue material parameter $\zeta_{t}$ controls the start of the slope shift.
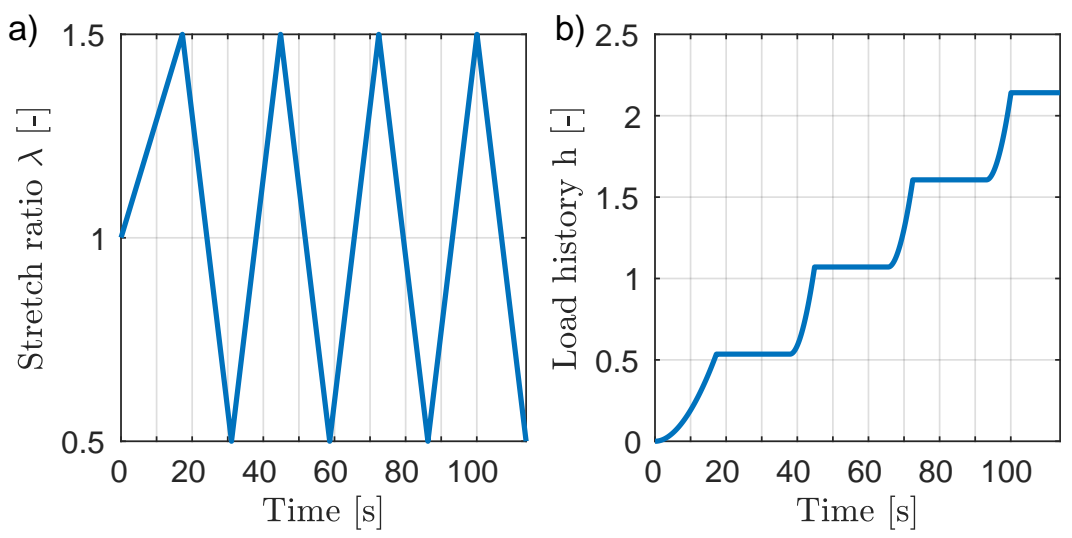

Figure B.25: Uniaxial tension-compression: a) Stretch-ratio versus time. b) Load history versus time. A compressive load does not increase the load history. 

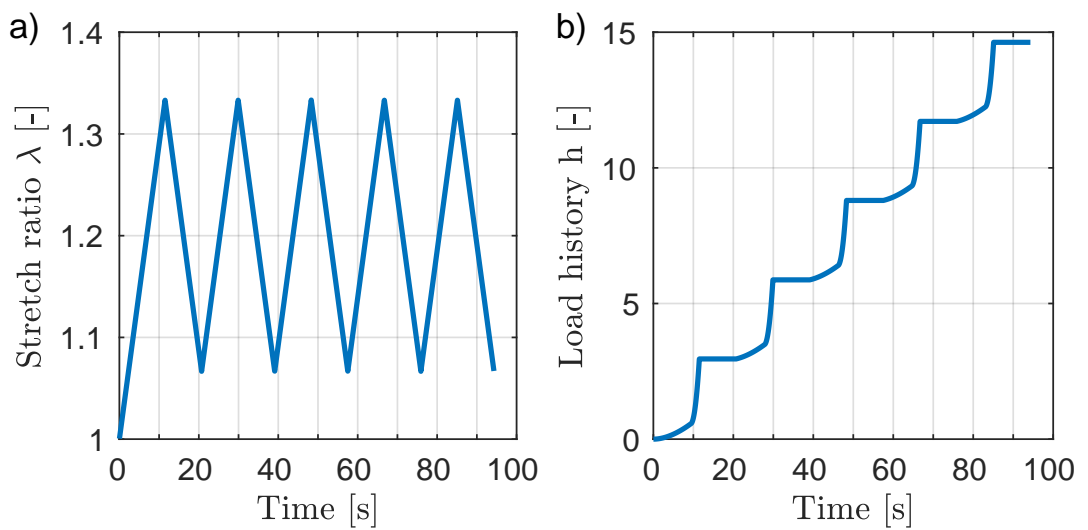

Figure B.26: Equibiaxial tension: a) Stretch-ratio versus time. b) Load history versus time. The load history grows faster after reaching threshold $\zeta_{t}$.
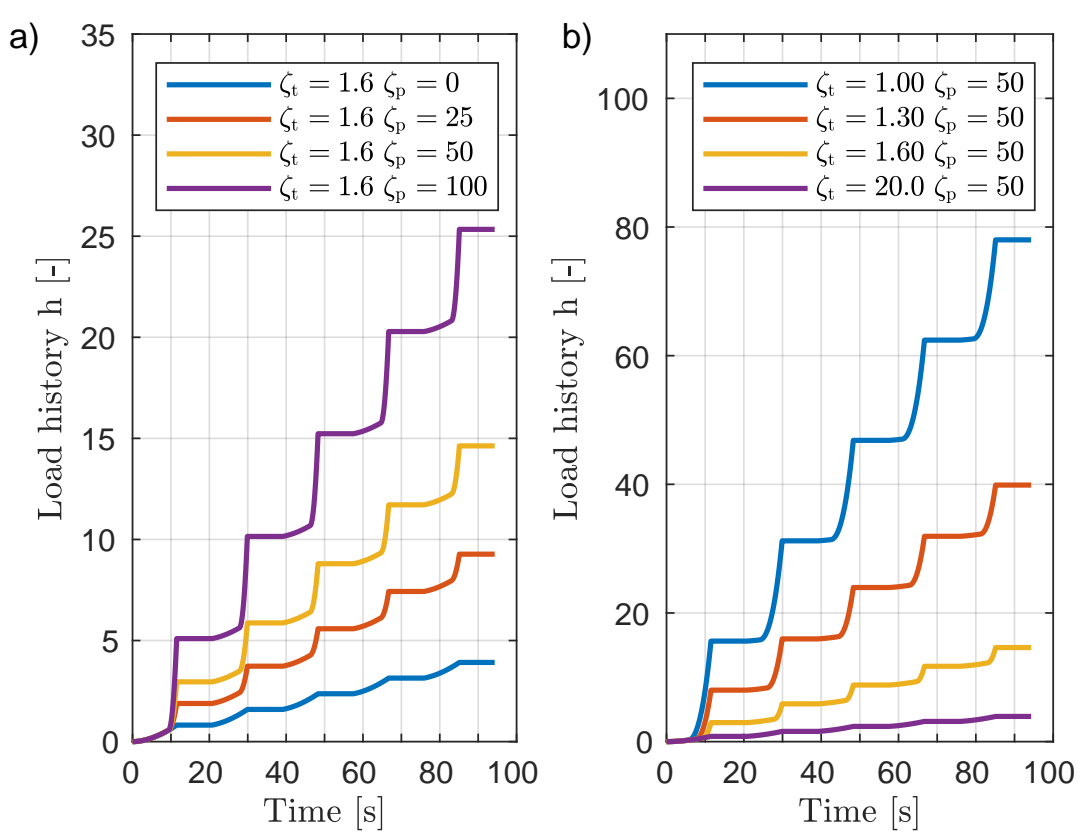

Figure B.27: Equibiaxial tension: Load history versus time for different values of a) $\zeta_{p}$ and b) $\zeta_{t}$. 


\section{Appendix C. Balance of mechanical energy and dissipation during crack growth}

We apply uniaxial cyclic tension to a bar (length $20 \mathrm{~mm}$, width $10 \mathrm{~mm}$ and thickness $1 \mathrm{~mm}$ ) and control the development of the energy of the various model components. The material parameters from Tabs. 4 5and 6 are used.

Fig. C.28 displays the applied stretch ratio versus time and we see an increasing history variable (Fig. C.28b). No damage occurs after 10 load cycles so that $D^{\text {crack }}=R=0$. The elastically stored energy and the applied external energy are in balance (Fig. C.28 ). Therefore, we change material parameters $a=50$ and $\beta_{d}=10^{3} \cdot \beta_{d}$ and present the respective results in Fig. C.29. Damage starts to grow with the first cycle, so that the elastically stored energy decreases and $R$ increases.

Finally, we conclude from Figs. C.28 and C.29 that the model is thermodynamically consistent since the balance of mechanical energy (Eq. (1)) is fulfilled at any given time. 

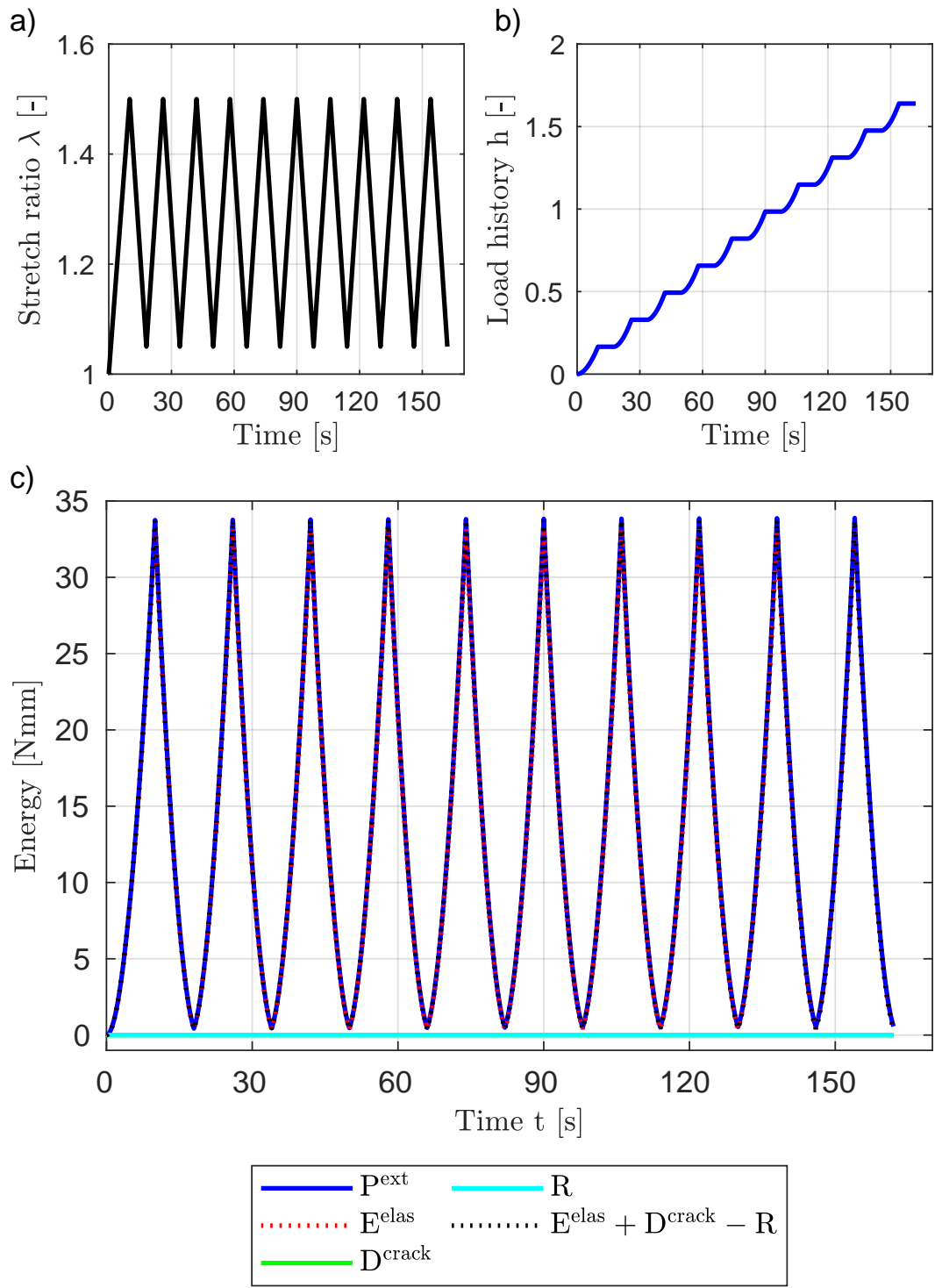

Figure C.28: Uniaxial cyclic tension: a) Stretch-ratio versus time. b) Load history versus time. c) Energy versus time for the different components of the model. The balance of mechanical energy (Eq. 11) is satisfied for the entire time. 

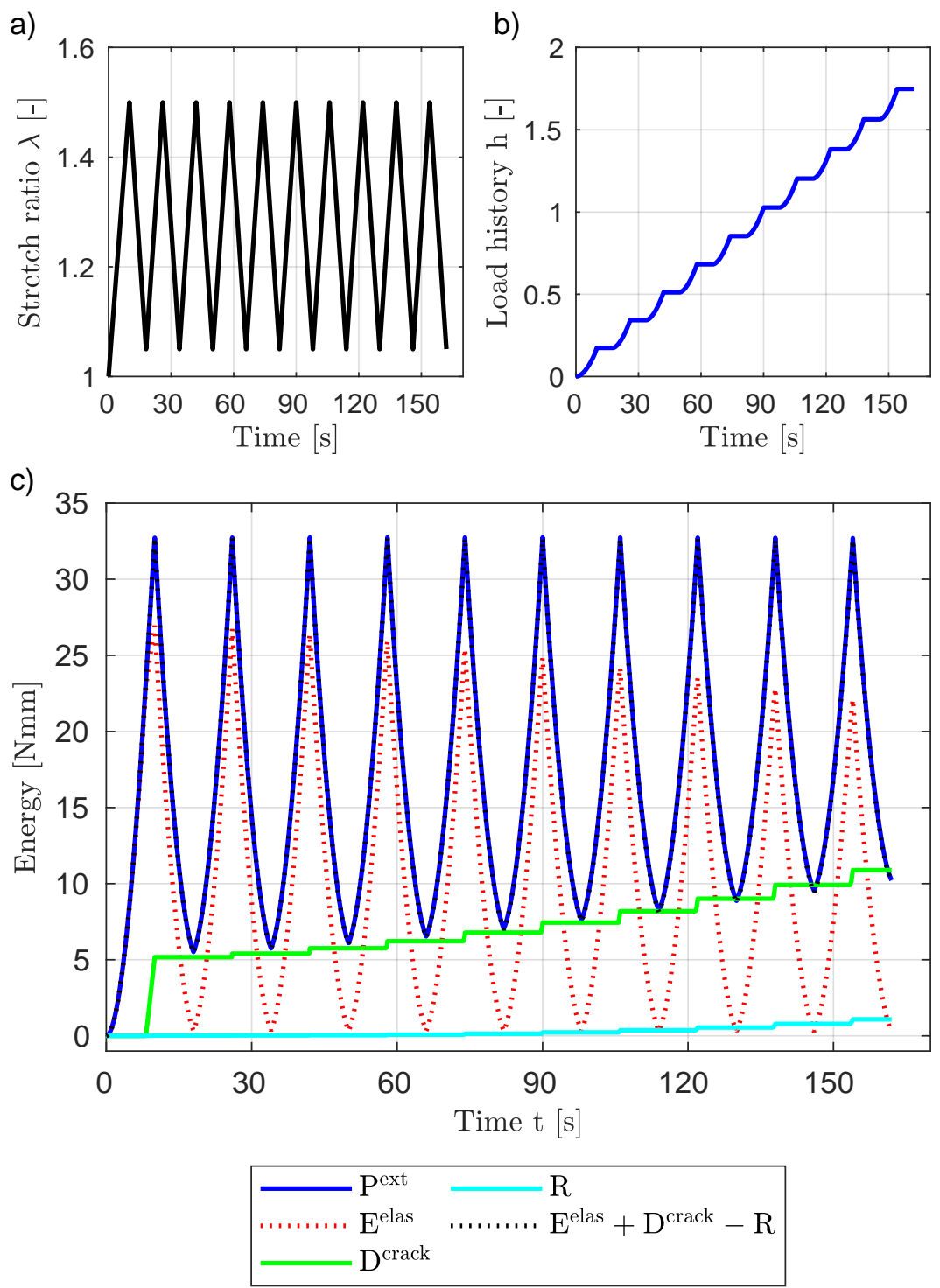

Figure C.29: Uniaxial cyclic tension: Material parameters $a=50$ and $\beta_{d}=10^{3} \cdot \beta_{d}$. a) Stretch-ratio versus time. b) Load history versus time. c) Energy versus time for the different components of the model. The balance of mechanical energy (Eq. (1)) is satisfied for the entire time. 


\section{Appendix D. Uniaxial compression}

We introduced in Eq. 28) an energy split for incompressible materials in plane stress to avoid damage growth in compression and crack surface penetration. In this appendix, the split is evaluated with two test cases. All examples in this section use the material from Tabs. 1, 2 and 3, except that we reduce $G_{c}=0.5 \mathrm{~N} / \mathrm{mm}$ to enable faster damage growth.

First, we compress the specimen depicted in Fig. D.30 . Without the split, we observe a failure of the part (blue line, Fig. D.31) while using the split ensures that no damage occurs (red line).

The next test considers compression and tension of a sample with an initial crack (Fig. D.30p). The crack is introduced by initializing the model with $d=1$. Using the proposed split, the sample absorbs compressive forces but is not able to transfer tensile loads (Fig. D.31p).

We conclude that the split successfully prevents both damage to grow during compression as well as crack surface penetration. Note that (resistance-free) sliding of the crack surfaces in the plane of the crack cannot be captured accurately.
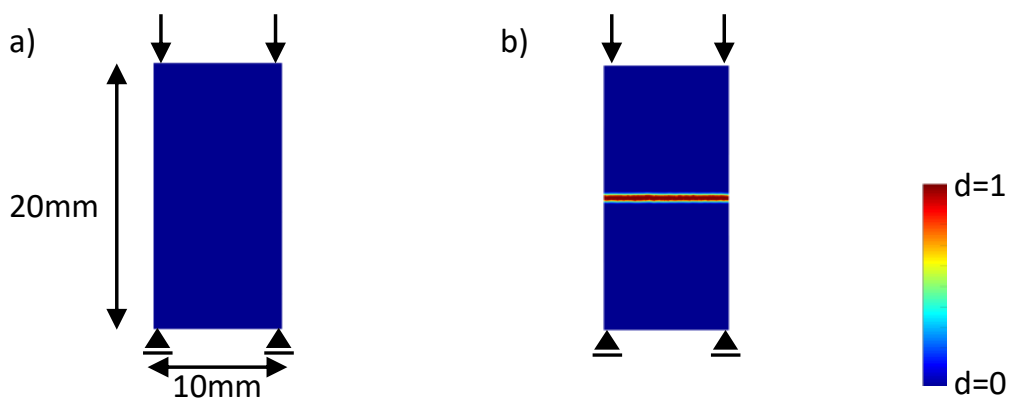

Figure D.30: Uniaxial compression: a) Dimensions and boundary conditions. b) A crack is introduced by initializing the model with $d=1$. 

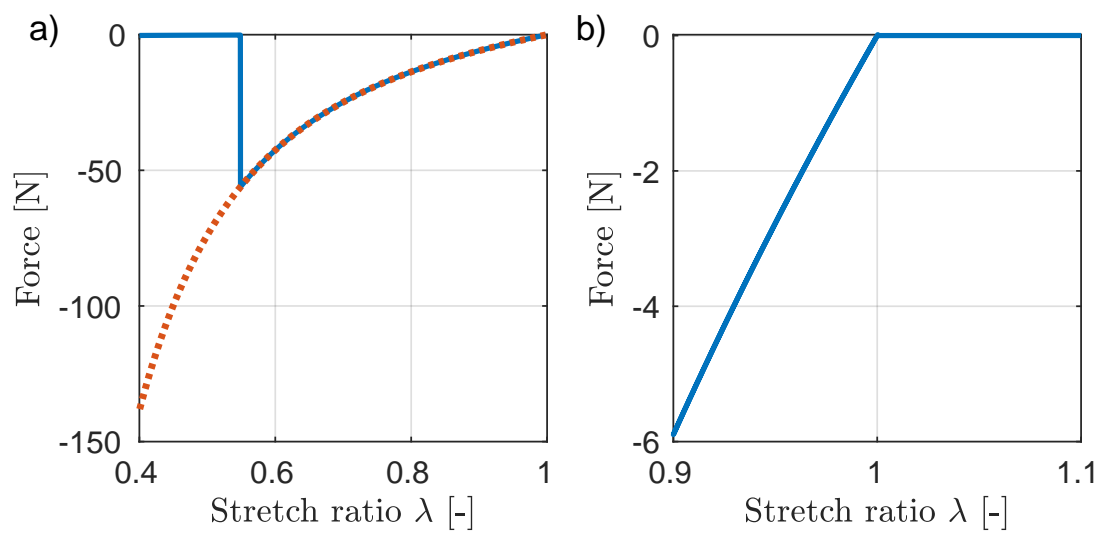

Figure D.31: Uniaxial compression: Force versus stretch ratio: a) Compression with the split (Eq. 28 red line) and without (blue line). b) Compression and tension of a sample with an initial crack. 


\section{References}

[1] P. J. Loew, B. Peters, and L. A. A. Beex. Fatigue phase-field damage modeling of rubber using viscous dissipation: Crack nucleation and propagation. Mechanics of Materials, 142, 2020.

[2] C. Miehe, M. Hofacker, and F. Welschinger. A phase field model for rateindependent crack propagation: Robust algorithmic implementation based on operator splits. Computer Methods in Applied Mechanics and Engineering, 199(45-48):2765-2778, 2010.

[3] C. Miehe, F. Welschinger, and M. Hofacker. Thermodynamically consistent phase-field models of fracture: Variational principles and multi-field FE implementations. International Journal for Numerical Methods in Engineering, 83(10):1273-1311, 2010.

[4] B. Bourdin, G. A. Francfort, and J. J. Marigo. Numerical experiments in revisited brittle fracture. Journal of the Mechanics and Physics of Solids, 48(4):797-826, 2000.

[5] V. P. Nguyen, O. Lloberas-Valls, M. Stroeven, and L. J. Sluys. Homogenization-based multiscale crack modelling: From micro-diffusive damage to macro-cracks. Computer Methods in Applied Mechanics and Engineering, 200:1220-1236, 2011.

[6] S. Wulfinghoff. A generalized cohesive zone model and a grain boundary yield criterion for gradient plasticity derived from surface- and interfacerelated arguments. International Journal of Plasticity, 92:57-78, 2017.

[7] L. A. A. Beex and R. H. J. Peerlings. An experimental and computational study of laminated paperboard creasing and folding. International Journal of Solids and Structures, 46(24):4192-4207, 2009.

[8] L. A. A. Beex and R. H. J. Peerlings. On the influence of delamination on laminated paperboard creasing and folding. Philosophical Transactions 
of the Royal Society A: Mathematical, Physical and Engineering Sciences, 370(1965):1912-1924, 2012.

[9] N. Moës, J. Dolbow, and T. Belytschko. A finite element method for crack growth without remeshing. International Journal for Numerical Methods in Engineering, 46(1):131-150, 1999.

[10] S. Bordas, V. P. Nguyen, C. Dunant, A. Guidoum, and H. Nguyen-Dang. An extended finite element library. International Journal for Numerical Methods in Engineering, 71(6):703-732, 2007.

[11] I. V. Singh, B. K. Mishra, S. Bhattacharya, and R. U. Patil. The numerical simulation of fatigue crack growth using extended finite element method. International Journal of Fatigue, 36(1):109-119, 2012.

[12] Y. Wang and H. Waisman. From diffuse damage to sharp cohesive cracks: A coupled XFEM framework for failure analysis of quasi-brittle materials. Computer Methods in Applied Mechanics and Engineering, 299:57-89, 2016.

[13] R. H. J. Peerlings, R. de Borst, W. A. M. Brekelmans, and J. H. P. de Vree. Gradient enhanced damage for quasi-brittle materials. International Journal for Numerical Methods in Engineering, 39(19):3391-3403, 1996.

[14] L. H. Poh and G. Sun. Localizing gradient damage model with decreasing interactions. International Journal for Numerical Methods in Engineering, 110(6):503-522, 2017.

[15] G. Sun and L. H. Poh. Homogenization of intergranular fracture towards a transient gradient damage model. Journal of the Mechanics and Physics of Solids, 95:374-392, 2016.

[16] C. Steinke, I. Zreid, and M. Kaliske. On the relation between phase-field crack approximation and gradient damage modelling. Computational Mechanics, 59(5):717-735, 2017. 
[17] R. de Borst and C. V. Verhoosel. Gradient damage vs phase-field approaches for fracture: Similarities and differences. Computer Methods in Applied Mechanics and Engineering, 312:78-94, 2016.

[18] T. K. Mandal, V. P. Nguyen, and A. Heidarpour. Phase field and gradient enhanced damage models for quasi-brittle failure: A numerical comparative study. Engineering Fracture Mechanics, 207:48-67, 2019.

[19] C. Miehe and L. M. Schänzel. Phase field modeling of fracture in rubbery polymers. Part I: Finite elasticity coupled with brittle failure. Journal of the Mechanics and Physics of Solids, 65(1):93-113, 2014.

[20] P. J. Loew, B. Peters, and L. A. A. Beex. Rate-dependent phase-field damage modeling of rubber and its experimental parameter identification. Journal of the Mechanics and Physics of Solids, 127:266-294, 2019.

[21] J. Wu, C. McAuliffe, H. Waisman, and G. Deodatis. Stochastic analysis of polymer composites rupture at large deformations modeled by a phase field method. Computer Methods in Applied Mechanics and Engineering, 2016.

[22] B. San and H. Waisman. Optimization of Carbon Black Polymer Composite Microstructure for Rupture Resistance. Journal of Applied Mechanics, 84(2):021005, 2016.

[23] J. B. Russ and H. Waisman. Topology optimization for brittle fracture resistance. Computer Methods in Applied Mechanics and Engineering, 347:238263, 2019 .

[24] G. Amendola, M. Fabrizio, and J. M. Golden. Thermomechanics of damage and fatigue by a phase field model. Journal of Thermal Stresses, 39(5):487499, 2016.

[25] M. Caputo and M. Fabrizio. Damage and fatigue described by a fractional derivative model. Journal of Computational Physics, 293:400-408, 2015. 
[26] J. L. Boldrini, E. A. Barros de Moraes, L. R. Chiarelli, F. G. Fumes, and M. L. Bittencourt. A non-isothermal thermodynamically consistent phase field framework for structural damage and fatigue. Computer Methods in Applied Mechanics and Engineering, 312:395-427, 2016.

[27] R. Alessi, S. Vidoli, and L. De Lorenzis. A phenomenological approach to fatigue with a variational phase-field model: The one-dimensional case. Engineering Fracture Mechanics, 190:53-73, 2018.

[28] P. Carrara, M. Ambati, R. Alessi, and L. De Lorenzis. A framework to model the fatigue behavior of brittle materials based on a variational phasefield approach. Computer Methods in Applied Mechanics and Engineering, 12731, 2019.

[29] A. Mesgarnejad, A. Imanian, and A. Karma. Phase-field models for fatigue crack growth. Theoretical and Applied Fracture Mechanics, 103, 2019.

[30] Y. S. Lo, M. J. Borden, K. Ravi-Chandar, and C. M. Landis. A phase-field model for fatigue crack growth. Journal of the Mechanics and Physics of Solids, 132, 2019.

[31] T. Heister, M. F. Wheeler, and T. Wick. A primal-dual active set method and predictor-corrector mesh adaptivity for computing fracture propagation using a phase-field approach. Computer Methods in Applied Mechanics and Engineering, 290:466-495, 2015.

[32] J. Cheng, X. Tu, and S. Ghosh. Wavelet-enriched adaptive hierarchical FE model for coupled crystal plasticity-phase field modeling of crack propagation in polycrystalline microstructures. Computer Methods in Applied Mechanics and Engineering, 361, 2019.

[33] R. U. Patil, B. K. Mishra, and I. V. Singh. A local moving extended phase field method (LMXPFM) for failure analysis of brittle materials. Computer Methods in Applied Mechanics and Engineering, 342:674-709, 2018. 
[34] P. Shanthraj, L. Sharma, B. Svendsen, F. Roters, and D. Raabe. A phase field model for damage in elasto-viscoplastic materials. Computer Methods in Applied Mechanics and Engineering, 312:167-185, 2016.

[35] J. Y. Wu, Y. Huang, and V. P. Nguyen. On the BFGS monolithic algorithm for the unified phase field damage theory. Computer Methods in Applied Mechanics and Engineering, 360, 2020.

[36] W. Van Paepegem and J. Degrieck. Fatigue degradation modelling of plain woven glass/epoxy composites. Composites - Part A: Applied Science and Manufacturing, 32(10):1433-1441, 2001.

[37] A. Turon, J. Costa, P. P. Camanho, and C. G. Dávila. Simulation of delamination in composites under high-cycle fatigue. Composites Part A: Applied Science and Manufacturing, 38(11):2270-2282, 2007.

[38] D. Cojocaru and A. M. Karlsson. A simple numerical method of cycle jumps for cyclically loaded structures. International Journal of Fatigue, 28(12):1677-1689, 2006.

[39] R. H. J. Peerlings, W. A. M. Brekelmans, R. De Borst, and M. G. D. Geers. Gradient-enhanced damage modelling of high-cycle fatigue. International Journal for Numerical Methods in Engineering, 49(12):1547-1569, 2000.

[40] C. Oskay and J. Fish. Fatigue life prediction using 2-scale temporal asymptotic homogenization. International Journal for Numerical Methods in Engineering, 61(3):329-359, 2004.

[41] J. Heczko and R. Kottner. Modeling of material damage using finite elements and time homogenization in case of finite strain. Applied Mathematics and Computation, 0:1-10, 2017.

[42] S. Manchiraju, M. Asai, and S. Ghosh. A dual-time-scale finite element model for simulating cyclic deformation of polycrystalline alloys. Journal of Strain Analysis for Engineering Design, 42(4):183-200, 2007. 
[43] D. S. Joseph, P. Chakraborty, and S. Ghosh. Wavelet transformation based multi-time scaling method for crystal plasticity FE simulations under cyclic loading. Computer Methods in Applied Mechanics and Engineering, 199(3336):2177-2194, 2010.

[44] M. Beusink. Multi-time scale methods for an efficient finite element analysis of fatigue problems. Master's thesis, Eindhoven University of Technology, 2013.

[45] M. E. Gurtin. Generalized Cahn-Hilliard equations based on a microforce balance. Physica, 2003(4):178-192, 1996.

[46] M. Frémond and B. Nedjar. Damage, gradient of damage and principle of virtual power. International Journal of Solids and Structures, 33(8):10831103, 1996.

[47] M. J. Borden, T. J. R. Hughes, C. M. Landis, A. Anvari, and I. J. Lee. A phase-field formulation for fracture in ductile materials: Finite deformation balance law derivation, plastic degradation, and stress triaxiality effects. Computer Methods in Applied Mechanics and Engineering, 312:130-166, 2016 .

[48] A. Kumar, G. A. Francfort, and O. Lopez-Pamies. Fracture and healing of elastomers: A phase-transition theory and numerical implementation. Journal of the Mechanics and Physics of Solids, 2018.

[49] H. Stumpf and K. Hackl. Micromechanical concept for the analysis of damage evolution in thermo-viscoelastic and quasi-brittle materials. International Journal of Solids and Structures, 40(6):1567-1584, 2003.

[50] J. Y. Wu and P. V. Nguyen. A length scale insensitive phase-field damage model for brittle fracture. Journal of the Mechanics and Physics of Solids, 119(l):20-42, 2018. 
[51] J. Y. Wu. A unified phase-field theory for the mechanics of damage and quasi-brittle failure. Journal of the Mechanics and Physics of Solids, 103:72-99, 2017.

[52] O. H. Yeoh. Some Forms of the Strain Energy Function for Rubber. Rubber Chemistry and Technology, 66(5):754-771, 2011.

[53] R. Shen, H. Waisman, and L. Guo. Fracture of viscoelastic solids modeled with a modified phase field method. Computer Methods in Applied Mechanics and Engineering, 346:862-890, 2019.

[54] D. Aranda-Iglesias, G. Vadillo, J. A. Rodríguez-Martínez, and K. Y. Volokh. Modeling deformation and failure of elastomers at high strain rates. Mechanics of Materials, 104:85-92, 2017.

[55] V. Le Saux, Y. Marco, S. Calloch, P. Charrier, and D. Taveau. Heat buildup of rubber under cyclic loadings: Validation of an efficient demarch to predict the temperature fields. Rubber Chemistry and Technology, 86(1):38$56,2013$.

[56] Y. Lev, A. Faye, and K. Y. Volokh. Thermoelastic deformation and failure of rubberlike materials. Journal of the Mechanics and Physics of Solids, 122:538-554, 2019.

[57] F. Stadlbauer, T. Koch, V. M. Archodoulaki, F. Planitzer, W. Fidi, and A. Holzner. Influence of experimental parameters on fatigue crack growth and heat build-up in rubber. Materials, 6(12):5502-5516, 2013.

[58] A. N. Gent. Engineering with Rubber: How to Design Rubber Components. Hanser Publishers, third edition, 2012.

[59] A. Cristiano, A. Marcellan, R. Long, C.-Y. Hui, J. Stolk, and C. Creton. An Experimental Investigation of Fracture by Cavitation of Model Elastomeric Networks. Journal of Polymer Science Part B: Polymer physics, 48:1409$1422,2010$. 
[60] A. Zine, N. Benseddiq, and M. Naït Abdelaziz. Rubber fatigue life under multiaxial loading: Numerical and experimental investigations. International Journal of Fatigue, 33(10):1360-1368, 2011.

[61] P. L. Rosendahl, M. Drass, J. Felger, J. Schneider, and W. Becker. Equivalent strain failure criterion for multiaxially loaded incompressible hyperelastic elastomers. International Journal of Solids and Structures, 166:32-46, 2019.

[62] M. Fassin, R. Eggersmann, S. Wulfinghoff, and S. Reese. Efficient algorithmic incorporation of tension compression asymmetry into an anisotropic damage model. Computer Methods in Applied Mechanics and Engineering, 354:932-962, 2019.

[63] J. Y. Wu, V. P. Nguyen, H. Zhou, and Y. Huang. A variationally consistent phase-field anisotropic damage model for fracture. Computer Methods in Applied Mechanics and Engineering, 358, 2020.

[64] H. Amor, J. J. Marigo, and C. Maurini. Regularized formulation of the variational brittle fracture with unilateral contact: Numerical experiments. Journal of the Mechanics and Physics of Solids, 57(8):1209-1229, 2009.

[65] G. A. Holzapfel. Nonlinear solid mechanics: A continuum approach for engineering. John Wiley \& Sons, second edition, 2001.

[66] J. Bonet and R. D. Wood. Nonlinear Continuum Mechanics for Finite Element Analysis. Cambridge University Press, 2008.

[67] J. Y. Wu and M. Cervera. A novel positive/negative projection in energy norm for the damage modeling of quasi-brittle solids. International Journal of Solids and Structures, 139-140:250-269, 2018.

[68] C. Steinke and M. Kaliske. A phase-field crack model based on directional stress decomposition. Computational Mechanics, 63(5):1019-1046, 2019. 
[69] H. Kiewel, J. Aktaa, and D. Munz. Application of an extrapolation method in thermocyclic failure analysis. Computer Methods in Applied Mechanics and Engineering, 182:55-71, 2000.

[70] A. Abdul-Latif, D. Razafindramary, and J. C. Rakotoarisoa. New hybrid cycle jump approach for predicting low-cycle fatigue behavior by a micromechanical model with the damage induced anisotropy concept. International Journal of Mechanical Sciences, 160:397-411, 2019.

[71] S. V. Hainsworth. An environmental scanning electron microscopy investigation of fatigue crack initiation and propagation in elastomers. Polymer Testing, 26(1):60-70, 2007. 